\title{
Design and Nonlinear Control of a 2-DOF Flexible Parallel Humanoid Arm Joint Robot
}

\author{
Leijie Jiang, Bingtuan Gao, and Zhenyu Zhu \\ School of Electrical Engineering, Southeast University, Nanjing 210096, China \\ Correspondence should be addressed to Bingtuan Gao; gaobingtuan@seu.edu.cn
}

Received 11 May 2017; Accepted 10 July 2017; Published 30 August 2017

Academic Editor: Evgeny Petrov

Copyright (C) 2017 Leijie Jiang et al. This is an open access article distributed under the Creative Commons Attribution License, which permits unrestricted use, distribution, and reproduction in any medium, provided the original work is properly cited.

\begin{abstract}
The paper focuses on the design and nonlinear control of the humanoid wrist/shoulder joint based on the cable-driven parallel mechanism which can realize roll and pitch movement. In view of the existence of the flexible parts in the mechanism, it is necessary to solve the vibration control of the flexible wrist/shoulder joint. In this paper, a cable-driven parallel robot platform is developed for the experiment study of the humanoid wrist/shoulder joint. And the dynamic model of the mechanism is formulated by using the coupling theory of the flexible body's large global motion and small flexible deformation. Based on derived dynamics, antivibration control of the joint robot is studied with a nonlinear control method. Finally, simulations and experiments were performed to validate the feasibility of the developed parallel robot prototype and the proposed control scheme.
\end{abstract}

\section{Introduction}

Live working is a better option for executing related tasks of the electric system. In consideration of the fact that traditional live working is mainly accomplished by the labour, it is dangerous and easy to cause personal casualty accidents. In order to avoid the accident in the artificial live working, it has urgent realistic meaning and important research value that the robot replaces the labour to execute live working. The traditional live working robot [1] has some drawbacks such as low ratio of load to mass and high insulation cost mainly due to the serial mechanism of the robot arm with embedded electrical driving parts, respectively. It is suggested to develop a self-insulated robot arm without embedded electric parts. Inspired by human arm's anatomical structure, the robot arm with cable-driven joints in parallel arrangement has already been studied [2]. According to the study, wrist/shoulder joint is the key module of the humanoid robot arm. Aiming at self-insulated requirement, the wrist/shoulder joint in [2] is simplified and redesigned as shown in Figure 1. The wrist/shoulder joint mechanism is in parallel arrangement with flexible spine and driven by three motors via three cables mimicking human muscles. Because of the existence of flexible parts in the mechanism which will cause vibration during motion control, it is necessary to study the problem of dynamics and vibration control for the wrist/shoulder joint. Figure 2 shows the developed robot prototype for the experimental study of the flexible wrist/shoulder joint mechanism in this paper.

Due to large workspace, high quality of load ratio, and small inertia, the cable-driven robot technology which stems from the crane system has become a research hot spot in the field of the robot $[3,4]$. The cable-driven parallel robots include the cable-driven planar robots and the cable-driven space robots. However, the cable-driven space robots are mostly cable-suspended parallel robots. The cable-driven parallel robots which have successful application have early RoboCrane [5] and SkyCam [6] which has an extensive application in television at present. A $500 \mathrm{~m}$ aperture spherical telescope (FAST) which performs the coarse positioning scheme by six driving cables is developed in China. Through the relevant research, the researchers obtain a series of research results $[7,8]$. Because the cables are soft and light and transmit the powerful action force, the cables are often used to actuate the bionic elephant-trunk [9] and the bionic cephalopods [10] which belong to cable-driven continuous/soft-bodied bionic robots [11].

The designed cable-driven parallel robot with a flexible spring belongs to the cable-driven parallel robot with an 


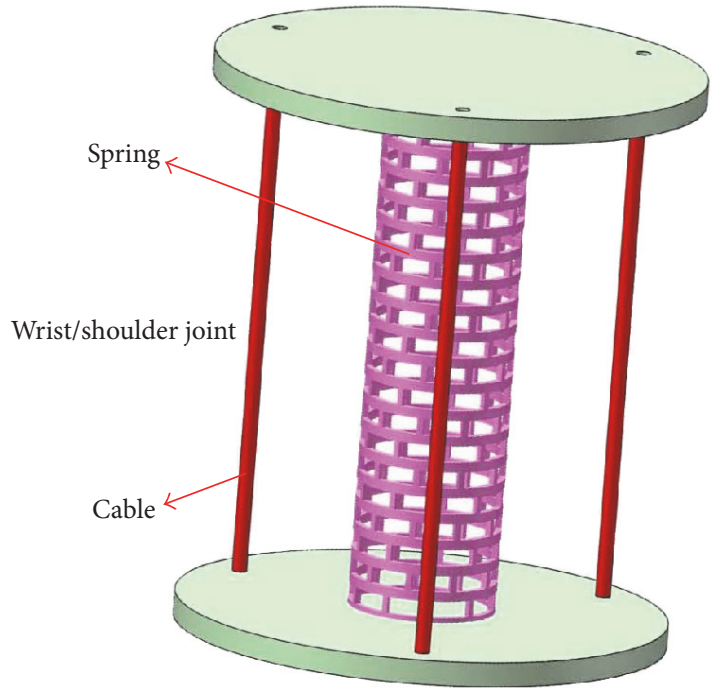

FIGURE 1: Structure design of the cable-driven wrist/shoulder joint.

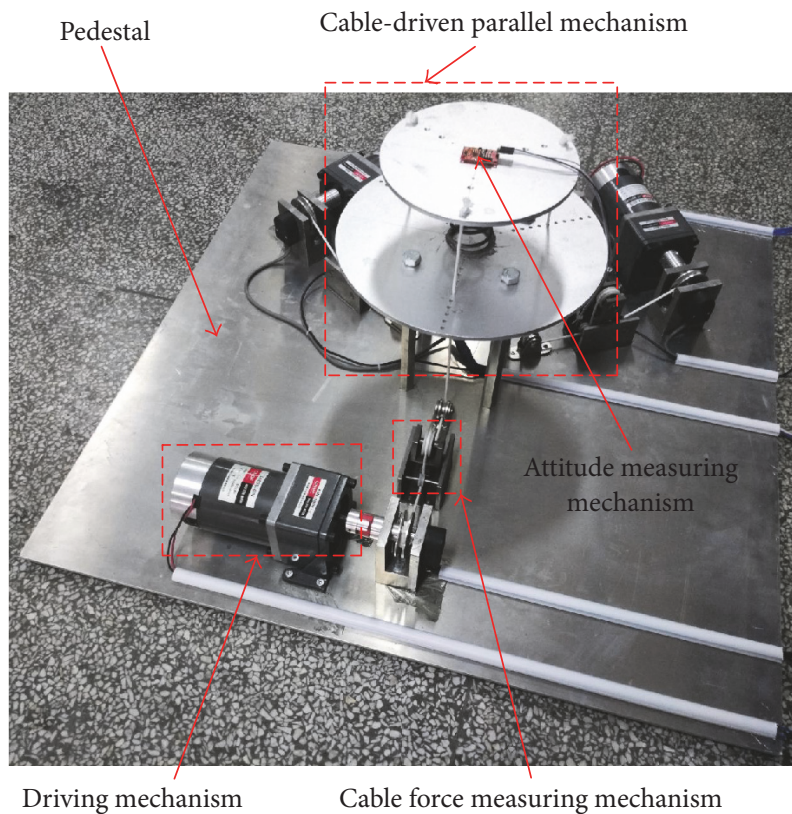

FIGURE 2: Prototype of the cable-driven parallel robot with a flexible spring.

upholder. Little research highlights how to design and control a robotic wrist/shoulder joint which moves smoothly like the people's wrist/shoulder joint. In light of the inspiration of people's wrist/shoulder joint structure, we elaborate a 2-DOF (roll and pitch) cable-driven parallel robot with a flexible spring. It includes a cable-driven parallel mechanism and the auxiliary mechanisms which consist of three guide mechanisms, four pillars, three driving mechanisms, a pedestal, an attitude measuring mechanism, three cable force measuring mechanisms, three cable length measuring mechanisms, and so on. The cable-driven parallel mechanism is the key mechanism of the proposed parallel robot. The spring replaces the articular bone in people's wrist/shoulder joint to support the moving platform and bends to one side to produce 2 -DOF (roll and pitch) movements. Three driving cables are equally spaced at $120^{\circ}$ on both the fixed base and the moving platform. The wrist/shoulder joint's 2-DOF (roll and pitch) movements are actuated by three cables which replace people's wrist/shoulder joint muscle. Three cables are pulled by three driving mechanisms mounted on the pedestal via three guide mechanisms on the pedestal.

Seeing that the proposed parallel robot has some flexible bodies such as the cylindrical compression spring and three cables, the moving platform must generate the flexible vibration during the parallel robot moves. In view of short length, light weight, and small diameter of the cables, cables are assumed to be linear elements that can only work in tension and the dynamical characteristics of cables themselves, such as the vibration and elongation, can be neglected [12,13]. As a result, the proposed parallel robot's vibration is mainly caused through the flexible cylindrical spring. Some researchers [14] studied the flexible system's kinematics and statics. However, they did not study the flexible body's vibration control problem. Therefore, we need to formulate a proper dynamical model of the parallel robot to establish a reasonable controller which can suppress the spring's vibration and enhance the parallel robot's location precision.

The issues of the flexible body's dynamics attract the researchers' attentions $[15,16]$. The dynamical modeling of the flexible body is usually formulated by the finite element method (FEM), the lumped mass method (LMM), and the assumed mode method (AMM). AMM has better computational efficiency and can establish more convenient control model than FEM and LMM. As a result, we establish the dynamic model of the parallel robot through AMM in this paper. Some researchers $[17,18]$ focus on the vibration control of the flexible mechanism. In [19], the research of Moallem et al. shows that the nonlinear control method based on inverse dynamics can effectively accomplish a trajectory tracking control with a small error of the flexible mechanism. Thus, we use nonlinear control method to design the vibration controller of the parallel robot in this paper.

The rest of this paper is organized as follows. In Section 2, the parallel robot design is presented. Then, the dynamic model of the parallel robot is established in Section 3. In Section 4, a nonlinear controller is designed based on the derived dynamical model. Next, the results of the simulation and the experiment are presented to validate the rationality of the robot prototype and the proposed control method in Section 5. Finally, conclusions and future work are summarized in Section 6.

\section{Robot Design}

This section will elaborate the parallel robot design which includes mechanical design and electrical design.

2.1. Mechanical Design. The solid model of the parallel robot is illustrated in Figure 3. As shown in Figures 2 and 3, the whole parallel robot mainly consists of the cable-driven 


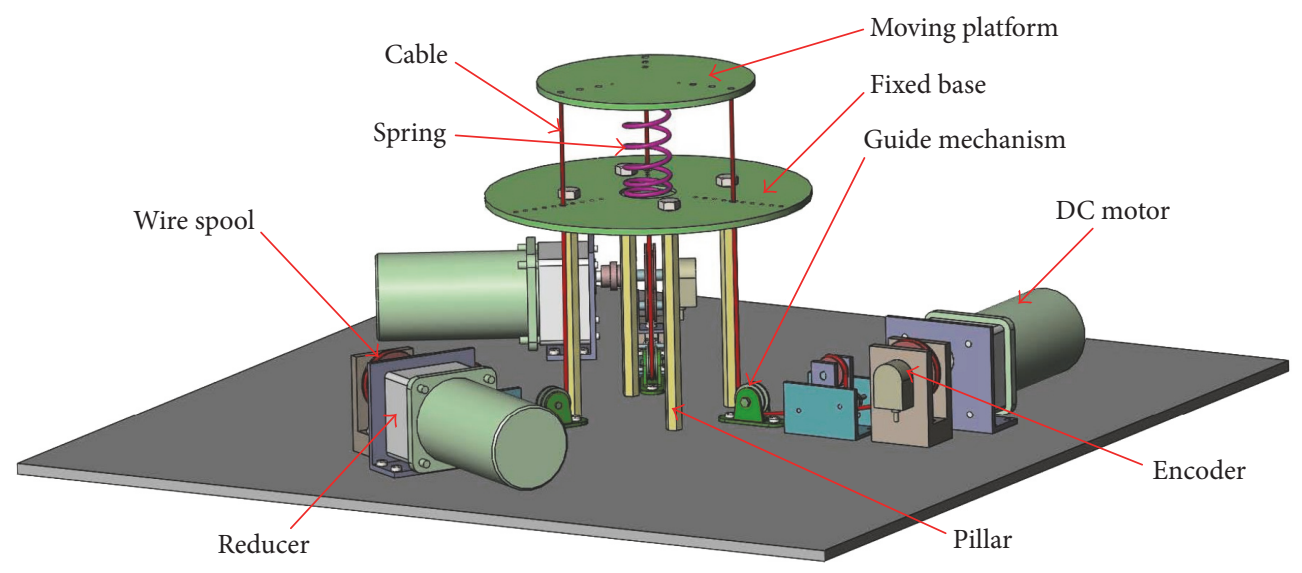

FIGURE 3: Solid model of the cable-driven parallel robot with a flexible spring.

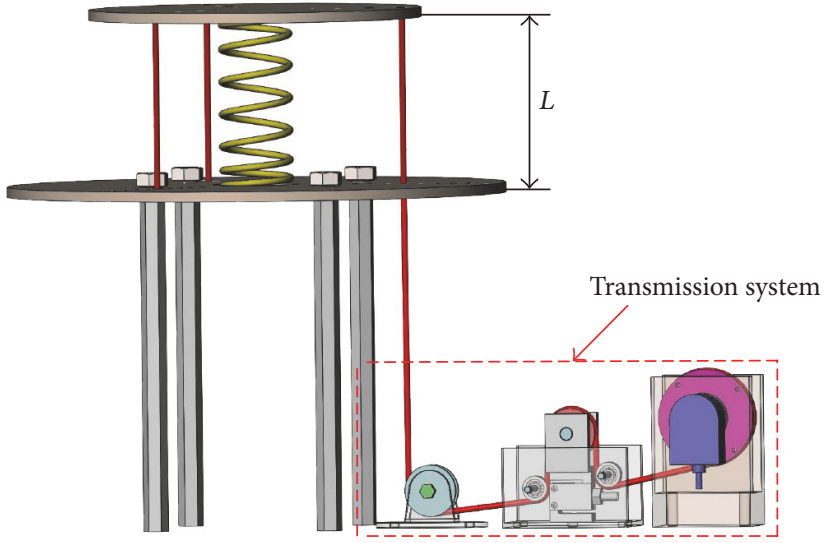

(a)

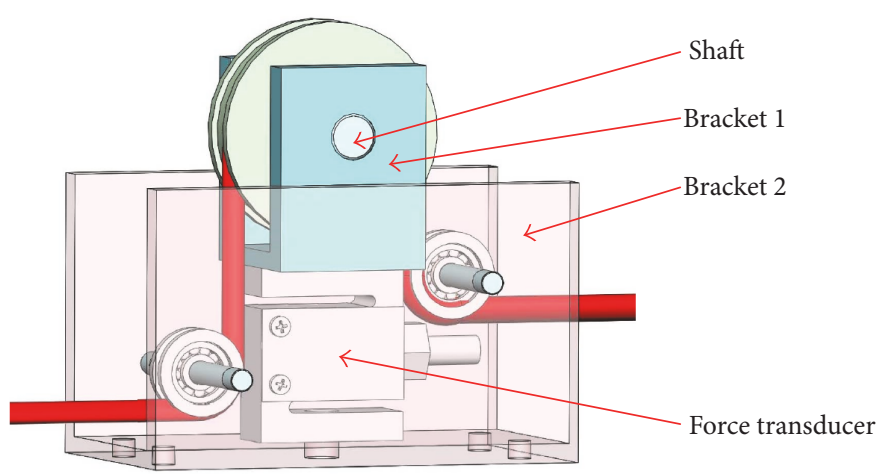

(b)

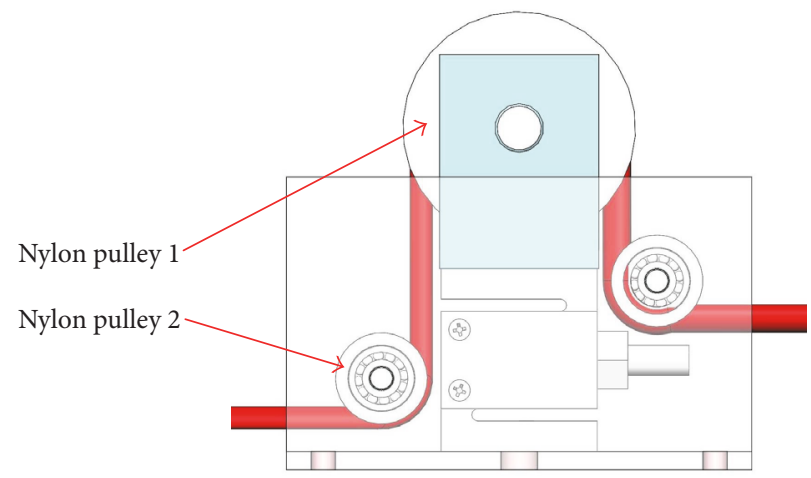

(c)

FIGURE 4: Local mechanical design of the parallel robot: (a) cable force transmission mechanism, (b) perspective 1 of the cable force measuring mechanism, and (c) perspective 2 of the cable force measuring mechanism.

parallel mechanism, the driving mechanism, and the cable force measuring mechanism. The local mechanical design of the parallel robot is depicted in Figure 4.

As shown in Figure 3, the cable-driven parallel mechanism consists of the moving platform, the fixed base, the flexible spring, and three cables. The aluminium alloy is applied in the moving platform and the fixed base. The cotton rope of $3 \mathrm{~mm}$ diameter is used as the cable material.
The top and the bottom of the helical compression spring are all planar. The moving platform and the fixed base are connected by the flexible spring. The power epoxy is used in the interfaces of the flexible spring and the moving platform and the fixed base; namely, the connection types between the spring and the moving platform and the fixed based are rigidjointed free end and rigid-jointed fixed end, respectively. Each cable and the moving platform are connected by the 
TABLE 1: Parameters of the DC gear motor.

\begin{tabular}{lcc}
\hline Parameter (units) & Value & Description \\
\hline$P_{r}(\mathrm{~W})$ & 40 & Rated power of DC motor \\
$U_{r}(\mathrm{~V})$ & 24 & Rated voltage of DC motor \\
$I_{r}(\mathrm{~A})$ & 2.1 & Rated current of DC motor \\
$n_{r}(\mathrm{r} / \mathrm{min})$ & 1800 & Rated speed of DC motor \\
$\hat{i}_{r}$ & $180: 1$ & Reduction ratio of the reducer \\
\hline
\end{tabular}

knot. The friction force between each cable and the fixed base is reduced using the fillet in each hole in the fixed base. The whole weight of the cable-driven parallel mechanism is supported by four pillars.

Each driving mechanism is made up of a direct current (DC) motor and a reducer. The parameters of the DC gear motor are given in Table 1 . The assembly diagram of the DC motor and the reducer is shown in Figure 3.

Each cable force is a very important measurand in cablebased architectures. In order to perform a measurement, a low-cost mechanism is presented for the cable force measuring in the proposed parallel robot. The mechanical design of the cable force measuring mechanism is illustrated in Figures 4(b) and 4(c). The cable force measuring mechanism mainly includes the nylon pulleys, the brackets, and the force transducer. Each cable's winding direction is shown in Figure 4(c). The cables between nylon pulley 1 and nylon pulley 2 on the force transducer's both sides are all vertical. $T_{c}$ denotes the cable force at a given time $t_{e}, W$ denotes the gross weight of the bracket 1 , nylon pulley 1 , and the shaft, and $F$ represents the force transducer's measuring value at a given time $t_{e}$. We assume that the cable's deformation, the cable's weight, and the friction force between the cable and the nylon pulleys can be neglected. Therefore, $T_{c}$ can be given as

$$
T_{c}=\frac{1}{2}(F-W) .
$$

Each cable length is also such an important measurand in cable-based architectures. To perform a measurement, a simple mechanism is presented for the cable length measuring in the proposed parallel robot. The cable transmission system is depicted in Figure 4(a). $n_{e}$ is the resolution of the incremental encoder. $p_{e}$ is the number of pulses, output for the incremental encoder at a given time $t_{e} . d_{s}$ is the diameter of the wire spool. $L$ denotes the original length of the spring. If we neglect the deformation of the cable, the cable length $l_{c}$ between the moving platform and the fixed base at a given time $t_{e}$ can be expressed as

$$
l_{c}=L-\frac{\pi d_{s} p_{e}}{n_{e}} .
$$

For such a cable force transmission system which is shown in Figure 4(a), the relation of $T_{c}$ and the armature current of the motor $\left(I_{a}\right)$ at a given time $t_{e}$ is certain. The detailed process of derivation of the theoretical relationship is as follows.
The DC motor's electromagnetic torque $\widehat{T}_{e}$ at a given time $t_{e}$ is given as

$$
\widehat{T}_{e}=C_{T} \Phi I_{a}
$$

where $C_{T}$ denotes the DC motor's torque constant and $\Phi$ denotes the DC motor's main magnetic flux.

If we neglect the DC motor's friction torque and the reducer's friction torque, the reducer's output torque $\widehat{T}_{r}$ at a given time $t_{e}$ can be expressed as

$$
\widehat{T}_{r}=\widehat{i}_{r} \widehat{T}_{e} .
$$

$T_{c}$ is given as

$$
T_{c}=\frac{2 \widehat{T}_{r}}{d_{s}} .
$$

Based on (3), (4), and (5), $T_{c}$ can be expressed as

$$
T_{c}=\frac{2 \hat{i}_{r} C_{T} \Phi I_{a}}{d_{s}} .
$$

Considering that $\hat{i}_{r}, C_{T}, \Phi$, and $d_{s}$ are all constant, $T_{c}$ and $I_{a}$ at a given time $t_{e}$ are in a directly proportional relationship.

2.2. Electrical Design. The architecture of the control system for the parallel robot is shown in Figure 5. The whole control system mainly consists of six parts: the main computer, the motion control card, the data acquisition card, the sensors, the motor drivers, and the power supply modules.

The motion control card (USB9010) which is powered by the main computer can control three motor drivers through the analog output mode and collect the data from three incremental encoders. The data acquisition card (USB 8AD) which is powered by the main computer can capture the data from three current sensors and three force transducers.

The sensors contain three incremental encoders, three current sensors, three force transducers, and an AHRS (attitude heading reference system) module. The resolution of each incremental encoder which is powered by the motion control card is 1000 lines per rev. The incremental encoder resolution denotes the output pulse number of the encoder during each rev. Each current sensor is powered by a switching power supply whose output voltage is $5 \mathrm{~V}$. Each force transducer is powered by a signal amplifier (DYBSQ-001) which can be powered by a switching power supply whose output voltage is $24 \mathrm{~V}$. The AHRS module contains a control chip (STM32F103T8), a MPU6050 (a triaxis accelerometer and a triaxis gyroscope), a HMC5883 (a triaxis magnetometer), and a BMP180 (an atmospheric pressure altimeter). The AHRS module which is powered by the main computer can measure the moving platform's posture.

Each motor driver which is powered by a switching power supply whose output voltage is $24 \mathrm{~V}$ can drive a DC motor through the torque control mode. The switching power supply can power the objects within the dashed box in Figure 5. 


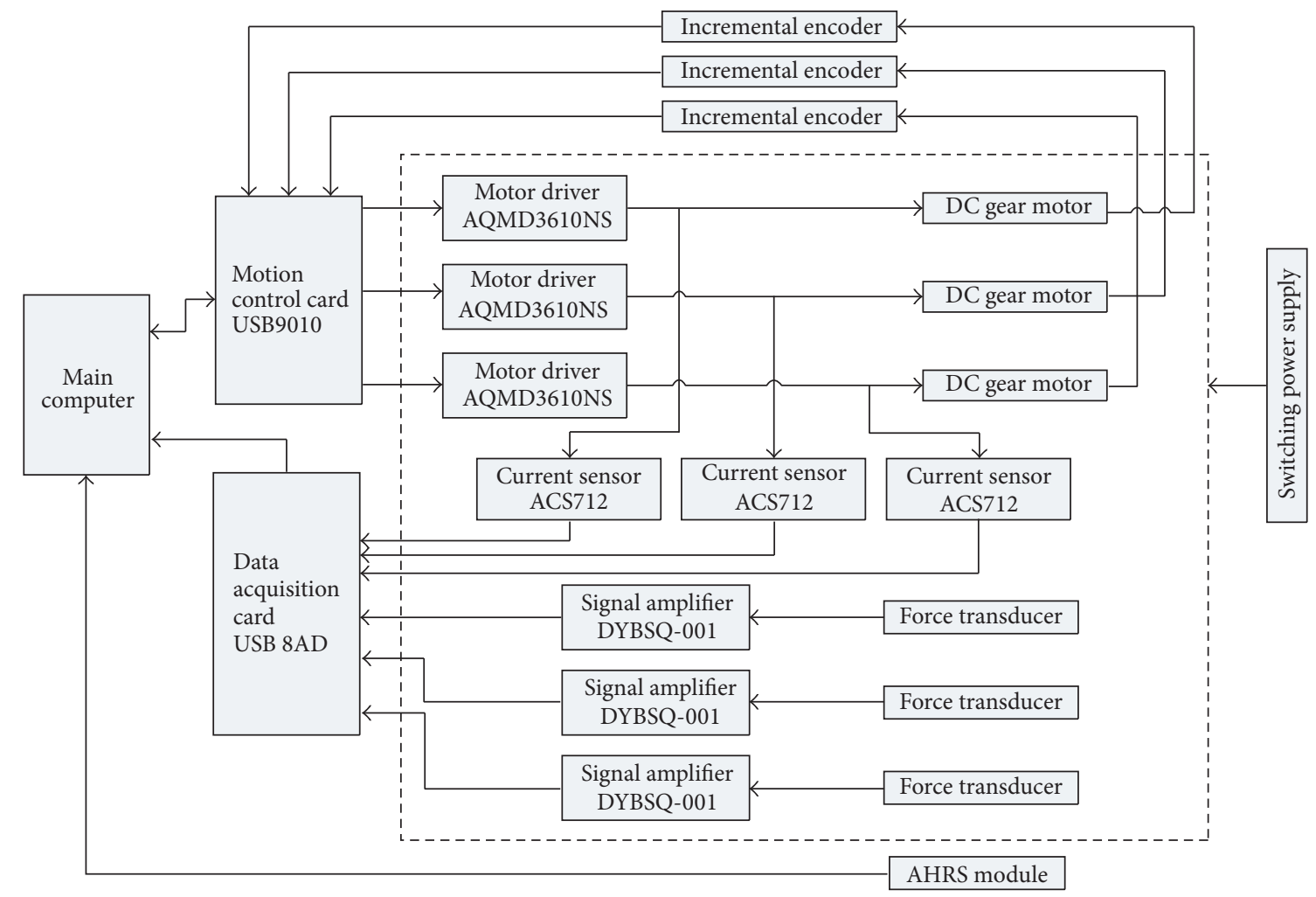

FIgURE 5: Architecture of the control system.

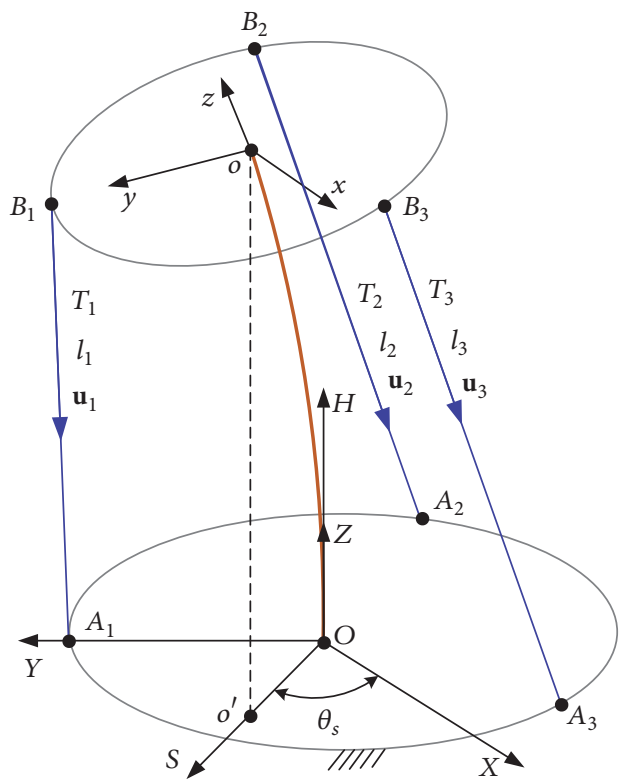

FiguRE 6: Schematic of the cable-driven parallel mechanism.

\section{Dynamics Model}

3.1. System Definition. This part illustrates the cable-driven parallel mechanism's schematic which is depicted in Figure 6. A local-coordinate system $o x y z$ is attached to the moving platform with origin $o$ located at its center. The $y$-axis is along the vector $\overrightarrow{o B_{1}}$. A global-coordinate system $O X Y Z$ whose origin $O$ is located at the bottom center of the flexible spring is attached to the fixed base. The $Y$-axis is along the vector $\overrightarrow{O A_{1}}$. The crooked flexible spring which is denoted by the curve Oo can manufacture a force/torque to sustain the moving platform. Three cables whose diameter size and mass can be neglected are connected to the points $B_{i}(i=1,2,3)$ which are located at the moving platform and pulled by three DC motors from the points $A_{i}(i=$ $1,2,3)$ which are located at the fixed base. The points $B_{i}$ and $A_{i}$ are located at equal arc length on both the moving platform and the fixed base, respectively. The moving platform's radius is $\left|\overrightarrow{O B_{i}}\right|=b \cdot\left|\overrightarrow{O A_{i}}\right|=a$ is the fixed base's radius. The homogeneous coordinates of $B_{i}$ in the localcoordinate system oxyz can be described, respectively, as ${ }^{o} \mathbf{b}_{1}=\left(\begin{array}{llll}0 & b & 0 & 1\end{array}\right)^{\mathrm{T}},{ }^{o} \mathbf{b}_{2}=\left(\begin{array}{llll}-(\sqrt{3} / 2) b & -(1 / 2) b & 0 & 1\end{array}\right)^{\mathrm{T}}$, and ${ }^{o} \mathbf{b}_{3}=((\sqrt{3} / 2) b \quad-(1 / 2) b \quad 0 \quad 1)^{\mathrm{T}}$; the homogeneous coordinates of $A_{i}$ in the global-coordinate system OXYZ can be described, respectively, as $\mathrm{O}_{\mathbf{a}_{1}}=$ $\left(\begin{array}{llll}0 & a & 0 & 1\end{array}\right)^{\mathrm{T}},{ }^{\mathrm{O}} \mathbf{a}_{2}=\left(\begin{array}{llll}-(\sqrt{3} / 2) a & -(1 / 2) a & 0 & 1\end{array}\right)^{\mathrm{T}}$, and

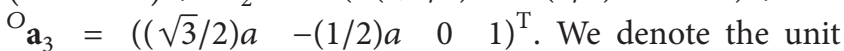
vector along the vectors $\overrightarrow{B_{i} A_{i}}(i=1,2,3)$ as $\mathbf{u}_{i}(i=1,2,3)$, the norm of the vectors $\overrightarrow{B_{i} A_{i}}(i=1,2,3)$ as $l_{i}(i=1,2,3)$, and the cable force as $T_{i}(i=1,2,3)$.

The flexible spring is supposed to crook in the same lateral plane and the moving platform will have no torsional behavior with respect to the $z$-axis in the local-coordinate 


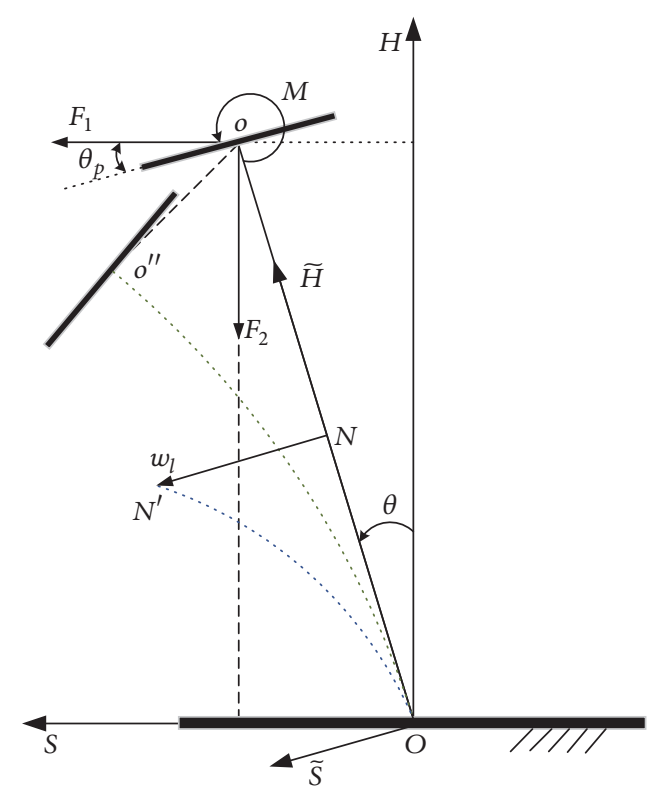

FiguRE 7: Deformation principle and force equivalent.

system oxyz. Moreover, a rectangular coordinate system OSH is attached to the plane Ooo'. The $S$-axis is along the vector $\overrightarrow{\mathrm{Oo}^{\prime}}$. The $H$-axis is along the $Z$-axis. We denote the angle between the $X$-axis and the $S$-axis as $\theta_{s}$. We denote the three-dimensional coordinates of the point $o$ in the globalcoordinate system $O X Y Z$ as $\bar{x}, \bar{y}, \bar{z}$, the moving platform's rotation with respect to the $X$-axis as $\alpha$, and the moving platform's rotation with respect to the $Y$-axis as $\beta$. The orientation matrix of the local-coordinate system $o x y z$ with respect to the global-coordinate system $O X Y Z$ is described as

$$
\widehat{\mathbf{R}}=\left(\begin{array}{ccc}
\cos \beta & \sin \alpha \sin \beta & \cos \alpha \sin \beta \\
0 & \cos \alpha & -\sin \alpha \\
-\sin \beta & \sin \alpha \cos \beta & \cos \alpha \cos \beta
\end{array}\right)
$$

3.2. Dynamics Equation. The workspace analysis of the proposed flexible parallel humanoid arm joint robot is presented in [20]. In this paper, the deformation of lateral dynamic bend for the spring is described by using the coupling theory of the flexible body's large global motion and small flexible deformation. By trial and error method, we pick a suitable modal function to guarantee that the deformation of lateral dynamic bend for the spring is within the scope of the workspace of the proposed flexible parallel humanoid arm joint robot. Moreover, the spring's deformation belongs to millimeter level. Therefore, the following analysis for the spring is valid for small deformation. The deformation principle and force equivalent are illustrated in Figure 7. We denote an arbitrary point in the flexible spring $O N$ as $N$. In view of the short length of the flexible spring, we only consider the spring's transverse deformation displacement $w_{l}$. Due to the deformation, the straight spring $O N$ will become the crooked spring $O N^{\prime}$. The norm of the vector $\overrightarrow{N N^{\prime}}$ is equal to $\left|w_{l}\right|$. We denote the angular displacement of the crooked spring $O N$ as $\theta$ and the norm of the vector $\overrightarrow{O N}$ as $h$. The transverse deformation displacement of any point $N$ in the spring is described as $w_{l}(h, t)=\delta(h) \mathbf{d}(t) . \delta(h)=\left(\delta_{1}(h), \delta_{2}(h), \ldots, \delta_{n}(h)\right)$ denotes the transverse vibration's modal function of the flexible spring. In view of the theory of mechanical vibration, we can get a fact that the deformation of the flexible spring in Figure 7 is similar to the first-order modal deformation of the inverted vertical beam. Hence, the spring's deformation can be described through the coupling theory of the flexible body's large-scale motion and small deformation. In [21], the spring is regarded as Euler-Bernoulli beam which is used to model the transverse deformation. However, the simulation results show that the spring's deformation could be beyond the workspace of the proposed flexible parallel humanoid arm joint robot. Therefore, the modal function selection of the transverse deformation for the spring is unreasonable. As mentioned above, we give a relatively suitable modal function $\left(\delta_{i}(h)=\sin (i \pi h / 2 L), i=1,2, \ldots, n\right)$ after repeated correction and revision. $\mathbf{d}(t)=\left(d_{1}(t), d_{2}(t), \ldots, d_{n}(t)\right)^{\mathrm{T}}$ represents the transverse vibration's modal coordinate of the flexible spring.

The velocity of the point $N$ along the $\widetilde{S}$-axis can be given by $\widetilde{v}_{s}(N)=\dot{\theta} h+\dot{w}_{l}(h, t)$, where $\dot{\theta}$ denotes the first derivative of $\theta$ and $\dot{w}_{l}(h, t)$ represents the first derivative of $w_{l}(h, t)$.

The velocity of the point $N$ along the $\widetilde{H}$-axis can be given by $\widetilde{v}_{h}(N)=-\dot{\theta} w_{l}(h, t)$.

Therefore, the energy of the flexible spring which includes the kinetic energy $T_{s}$ and the potential energy $V_{s}$ is described as

$$
\begin{aligned}
& T_{s}=\frac{1}{2} \widehat{\rho}_{s} \int_{0}^{L}\left(\widetilde{v}_{s}^{2}(N)+\widetilde{v}_{h}^{2}(N)\right) \mathrm{d} h, \\
& V_{s}=\frac{1}{2} \int_{0}^{L} E I\left(\frac{\partial^{2} w_{l}(h, t)}{\partial h^{2}}\right)^{2} \mathrm{~d} h,
\end{aligned}
$$

where $\hat{\rho}_{s}$ represents the flexible spring's linear density, $E$ represents the flexible spring's modulus of elasticity, and I denotes the flexible spring's cross-sectional moment of inertia.

The energy of the moving platform containing the kinetic energy $T_{d}$ and the potential energy $V_{d}$ is expressed as

$$
\begin{aligned}
& T_{d}=\frac{1}{2} m_{d}\left(\widetilde{v}_{s}^{2}(o)+\widetilde{v}_{h}^{2}(o)\right)+\frac{1}{2} \widehat{\boldsymbol{\omega}}^{\mathrm{T}} \widehat{\mathbf{R}} \widehat{\mathbf{R}}^{\mathrm{T}} \widehat{\boldsymbol{\omega}}, \\
& V_{d}=-m_{d} g(L-L \cos \theta),
\end{aligned}
$$

where $m_{d}$ denotes the moving platform's mass, $\widehat{\boldsymbol{\omega}}=(\dot{\alpha}, \dot{\beta}, 0)^{\mathrm{T}}$, $\widetilde{v}_{h}(o)=-\dot{\theta} w_{l}(L, t)$ denotes the velocity of the point $o$ along the $\widetilde{H}$-axis, $\widetilde{v}_{s}(o)=\dot{\theta} L+\dot{w}_{l}(L, t)$ denotes the velocity of the point $o$ along the $\widetilde{S}$-axis, $g$ is the acceleration of gravity and 
is equal to $9.8 \mathrm{~m} / \mathrm{s}^{2}$, and $\widehat{\mathbf{I}}$ represents the moving platform's inertia moment, and it can be described as

$$
\widehat{\mathbf{I}}=\left(\begin{array}{ccc}
\frac{1}{4} m_{d} b^{2} & 0 & 0 \\
0 & \frac{1}{4} m_{d} b^{2} & 0 \\
0 & 0 & \frac{1}{2} m_{d} b^{2}
\end{array}\right) .
$$

Based on (8) and (10), the parallel robot system's kinetic energy is $T=T_{s}+T_{d}$; based on (9) and (11), the parallel robot system's potential energy is $V=V_{s}+V_{d}$.

As described in Figure 7, because of the spring's flexibility, $o$ point will run to $o^{\prime \prime}$ point at a given time. We denote the angle between the horizontal line and the moving platform as $\theta_{p}$ at a given time. Because the flexible spring is always assumed to crook in the lateral plane $O S H$, the effect of all cable forces is equal to the effect of the moment $M$ which is vertical to the lateral plane $O S H$ and the forces $F_{1}$ and $F_{2}$ which are vertical to each other in the lateral plane OSH. We hypothesize that the vector $\overrightarrow{\mathrm{Oo}}$ and the moving platform are always orthogonal; hence we have an inference of $\theta_{p}=\theta$. Considering that the potential energy of gravity of the moving platform has been taken into consideration in the system, the effect of gravity is overlooked. Hence we have

$$
\begin{aligned}
& \sum_{i=1}^{3} T_{i}^{O} \mathbf{u}_{i}=\left(\begin{array}{llll}
F_{1} \cos \theta_{s} & F_{1} \sin \theta_{s} & -F_{2}
\end{array}\right)^{\mathrm{T}}, \\
& \sum_{i=1}^{3}{ }^{O} \mathbf{r}_{i} \times T_{i}^{O} \mathbf{u}_{i}=\left(\begin{array}{lll}
-M \sin \theta_{s} & M \cos \theta_{s} & 0
\end{array}\right)^{\mathrm{T}},
\end{aligned}
$$

where ${ }^{O} \mathbf{u}_{i}=\left({ }^{O} \mathbf{a}_{i}-\widehat{\mathbf{T}}_{t}^{o} \mathbf{b}_{i}\right) /{ }^{O} \mathbf{a}_{i}-\widehat{\mathbf{T}}_{t}^{o} \mathbf{b}_{i} \mid$ and ${ }^{O} \mathbf{r}_{i}=\widehat{\mathbf{R}} \cdot \overrightarrow{o B_{i}}$; we denote the homogeneous transformation matrix of the localcoordinate system $o x y z$ with respect to the global-coordinate system $O X Y Z$ as $\widehat{\mathbf{T}}_{t}$, which can be expressed as

$$
\widehat{\mathbf{T}}_{t}=\left(\begin{array}{cccc}
\cos \beta & \sin \alpha \sin \beta & \cos \alpha \sin \beta & \bar{x} \\
0 & \cos \alpha & -\sin \alpha & \bar{y} \\
-\sin \beta & \sin \alpha \cos \beta & \cos \alpha \cos \beta & \bar{z} \\
0 & 0 & 0 & 1
\end{array}\right),
$$

where $\bar{x}=L \sin \theta \cos \theta_{s}, \bar{y}=L \sin \theta \sin \theta_{s}$, and $\bar{z}=L \cos \theta$.

We denote $o^{\prime \prime}$ point's spatial coordinates in the globalcoordinate system $O X Y Z$ as $x^{\prime}, y^{\prime}, z^{\prime}$. The bending moment of the flexible spring in the point $o^{\prime \prime}$ is denoted as $M_{o^{\prime \prime}}$, which is given as $M_{o^{\prime \prime}}=M+F_{1}\left(\bar{z}-z^{\prime}\right)+F_{2}\left(\sqrt{x^{\prime 2}+y^{\prime 2}}-\sqrt{\bar{x}^{2}+\bar{y}^{2}}\right)$, where $\bar{z}-z^{\prime}=\bar{e}_{1} \approx \int_{0}^{L} w_{l}(h, t) \sin \theta \mathrm{d} h$ and $\sqrt{x^{\prime 2}+y^{\prime 2}}-$ $\sqrt{\bar{x}^{2}+\bar{y}^{2}}=\bar{e}_{2} \approx \int_{0}^{L} w_{l}(h, t) \cos \theta \mathrm{d} h$.

In conclusion, the system's Lagrange function is given as $\bar{L}=T-V=T_{s}+T_{d}-V_{s}-V_{d}$. As previously mentioned, the lateral bending deformation of the flexible spring is described by the coupling theory of the flexible body's large overall motion and small deformation. Hence, the crooked spring's angular displacement $\theta$ and the transverse deformation's modal coordinate of the spring $\mathbf{d}(t)$ are selected as the generalized coordinate of the system. We denote the system's generalized coordinate as $\eta=(\theta \mathbf{d}(t))^{\mathrm{T}}$ and the system's generalized force as $\mathbf{Q}=\left(\begin{array}{ll}M_{o^{\prime \prime}} & \mathbf{0}_{n \times 1}\end{array}\right)^{\mathrm{T}}$. Therefore, the system's Lagrangian equation can be expressed as

$$
\frac{d}{d t}\left(\frac{\partial \bar{L}}{\partial \dot{\eta}}\right)-\frac{\partial \bar{L}}{\partial \eta}=\mathbf{Q} .
$$

We denote the generalized mass matrix as $\mathbf{M}$, Coriolis and centripetal term as $\mathbf{H}(\eta, \dot{\eta})$, the generalized stiffness matrix as $\mathbf{K}$, and the gravity term as $\mathbf{G}$. According to the above analysis, the dynamics equation of the system can be expressed as

$$
\mathbf{M} \ddot{\eta}+\mathbf{H}(\eta, \dot{\eta})+\mathbf{K} \eta+\mathbf{G}=\mathbf{Q} .
$$

In view of the existence of the system damping, we add Rayleigh viscous damping model $\mathbf{C}=\alpha \mathbf{M}+\beta \mathbf{K}$ to the system, where $\alpha$ and $\beta$ are Rayleigh damping scale coefficients. Therefore, the dynamics equation of the system is revised as

$$
\mathbf{M} \ddot{\eta}+\mathbf{C} \dot{\eta}+\mathbf{H}(\eta, \dot{\eta})+\mathbf{K} \eta+\mathbf{G}=\mathbf{Q}
$$

\section{Nonlinear Controller Design}

This part aims to design a nonlinear controller which can be used to track the position of the center for the moving platform while suppressing vibration of the flexible spring and to validate the effectiveness of the design for the cabledriven parallel robot and the proposed control strategy.

4.1. Nonlinear Control Model. Equation (17) can be expressed as

$$
\begin{gathered}
\left(\begin{array}{ll}
M_{11} & \mathbf{M}_{12} \\
\mathbf{M}_{21} & \mathbf{M}_{22}
\end{array}\right)\left(\begin{array}{l}
\ddot{\theta} \\
\ddot{\mathbf{d}}
\end{array}\right)+\mathbf{C}\left(\begin{array}{l}
\dot{\theta} \\
\dot{\mathbf{d}}
\end{array}\right)+\left(\begin{array}{l}
H_{1} \\
\mathbf{H}_{2}
\end{array}\right) \\
+\left(\begin{array}{ll}
K_{11} & \mathbf{K}_{12} \\
\mathbf{K}_{21} & \mathbf{K}_{22}
\end{array}\right)\left(\begin{array}{l}
\theta \\
\mathbf{d}
\end{array}\right)+\left(\begin{array}{l}
G_{1} \\
\mathbf{G}_{2}
\end{array}\right)=\left(\begin{array}{l}
M_{o^{\prime \prime}} \\
\mathbf{0}_{n \times 1}
\end{array}\right),
\end{gathered}
$$

where

$$
\begin{aligned}
\mathbf{M} & =\left(\begin{array}{ll}
M_{11} & \mathbf{M}_{12} \\
\mathbf{M}_{21} & \mathbf{M}_{22}
\end{array}\right), \\
\mathbf{H}(\eta, \dot{\eta}) & =\left(\begin{array}{l}
H_{1} \\
\mathbf{H}_{2}
\end{array}\right), \\
\mathbf{K} & =\left(\begin{array}{ll}
K_{11} & \mathbf{K}_{12} \\
\mathbf{K}_{21} & \mathbf{K}_{22}
\end{array}\right), \\
\mathbf{G} & =\left(\begin{array}{l}
G_{1} \\
\mathbf{G}_{2}
\end{array}\right),
\end{aligned}
$$

where the detailed expressions of $\mathbf{M}, \mathbf{H}(\eta, \dot{\eta}), \mathbf{K}$, and $\mathbf{G}$ are given in Appendix. 
Equation (18) is expressed as

$$
\mathbf{M}\left(\begin{array}{c}
\ddot{\theta} \\
\ddot{\mathbf{d}}
\end{array}\right)+\left(\begin{array}{c}
g_{1}(\theta, \dot{\theta}, \mathbf{d}, \dot{\mathbf{d}}) \\
\mathbf{g}_{2}(\theta, \dot{\theta}, \mathbf{d}, \dot{\mathbf{d}})+\mathbf{K}_{22} \mathbf{d}
\end{array}\right)=\left(\begin{array}{c}
\widehat{\mathbf{J}} \mathbf{T} \\
\mathbf{0}_{n \times 1}
\end{array}\right),
$$

where $\widehat{\mathbf{J} T}=M_{o^{\prime \prime}}, \widehat{\mathbf{J}}=\left(\begin{array}{lll}\widehat{J}_{1} & \widehat{J}_{2} & \widehat{J}_{3}\end{array}\right)$, and $\mathbf{T}=\left(\begin{array}{lll}T_{1} & T_{2} & T_{3}\end{array}\right)^{\mathrm{T}}$. The detailed expressions of $\widehat{\mathbf{J}}$ are given in Appendix.

Referring to (20), we define

$$
\mathbf{Z}=\mathbf{M}^{-1}=\left(\begin{array}{ll}
Z_{11} & \mathbf{Z}_{12} \\
\mathbf{Z}_{21} & \mathbf{Z}_{22}
\end{array}\right) .
$$

Multiplying (21) by both sides of (20), we have

$$
\begin{aligned}
& \ddot{\theta}=Z_{11}\left(M_{o^{\prime \prime}}-g_{1}\right)+Z_{12}\left(-\mathbf{g}_{2}-\mathbf{K}_{22} \mathbf{d}\right), \\
& \ddot{\mathbf{d}}=\mathbf{Z}_{21}\left(M_{o^{\prime \prime}}-g_{1}\right)+\mathbf{Z}_{22}\left(-\mathbf{g}_{2}-\mathbf{K}_{22} \mathbf{d}\right) .
\end{aligned}
$$

Our main goal is to achieve a small tracking error at the moving platform while suppressing the vibration in the flexible spring. To this end, we define the output as

$$
k=\theta+\frac{\delta(L) \mathbf{d}}{L} .
$$

As shown in Figure 7, (24) represents the angle between the straight line $O o^{\prime \prime}$ and the $H$-axis.

Substituting (22) and (23) into the second derivative of (24), we have

$$
\ddot{k}=B(\theta, \mathbf{d}, t) M_{o^{\prime \prime}}-c(\theta, \dot{\theta}, \mathbf{d}, \dot{\mathbf{d}}, t),
$$

where

$$
\begin{aligned}
B(\theta, \mathbf{d}, t)= & Z_{11}+\frac{\delta(L)}{L} \mathbf{Z}_{21}, \\
c(\theta, \dot{\theta}, \mathbf{d}, \dot{\mathbf{d}}, t)= & B(\theta, \mathbf{d}, t) g_{1} \\
& +\left(\mathbf{Z}_{12}+\frac{\delta(L)}{L} \mathbf{Z}_{22}\right)\left(\mathbf{g}_{2}+\mathbf{K}_{22} \mathbf{d}\right) .
\end{aligned}
$$

Let $M_{o^{\prime \prime}}$ take the following form:

$$
M_{o^{\prime \prime}}=B^{-1}(v+c) ;
$$

namely,

$$
\widehat{\mathbf{J}} \mathbf{T}=B^{-1}(v+c),
$$

where $v$ is a variable which depends on the desired output trajectory and the tracking error as defined later. We denote the desired output trajectory as $k_{d}$ and define

$$
\begin{aligned}
& e=k_{d}-k, \\
& \dot{e}=\dot{k}_{d}-\dot{k} .
\end{aligned}
$$

We choose

$$
v=\ddot{k}_{d}+C_{p} e+C_{d} \dot{e},
$$

where $C_{p}$ and $C_{d}$ are the control gains.
Substituting (28) into (25), we have

$$
\ddot{k}=v \text {. }
$$

Based on (31), (32), and (33), we have

$$
\dot{\Delta}=\mathbf{A}_{\Delta} \Delta,
$$

where

$$
\begin{aligned}
\Delta & =\left(\begin{array}{l}
e \\
\dot{e}
\end{array}\right), \\
\mathbf{A}_{\Delta} & =\left(\begin{array}{cc}
0 & 1 \\
-C_{p} & -C_{d}
\end{array}\right) .
\end{aligned}
$$

Based on (23), (27), and (28), we have

$$
\ddot{\mathbf{d}}=-\overline{\mathbf{P}} \mathbf{K}_{22} \mathbf{d}-\overline{\mathbf{P}} \mathbf{g}_{2}+\mathbf{Z}_{21} B^{-1} v,
$$

where

$$
\overline{\mathbf{P}}=\mathbf{Z}_{22}-\mathbf{Z}_{21} B^{-1}\left(\mathbf{Z}_{12}+\frac{\delta(L)}{L} \mathbf{Z}_{22}\right) .
$$

We define

$$
\Theta=\left(\begin{array}{l}
\mathbf{d} \\
\dot{\mathbf{d}}
\end{array}\right) .
$$

Based on (36), we have

$$
\dot{\Theta}=\widehat{\mathbf{A}} \Theta+\widehat{\mathbf{c}},
$$

where

$$
\begin{aligned}
& \widehat{\mathbf{A}}=\left(\begin{array}{cc}
\mathbf{0}_{n \times n} & \mathbf{I}_{n \times n} \\
-\overline{\mathbf{P}} \mathbf{K}_{22} & \mathbf{0}_{n \times n}
\end{array}\right), \\
& \widehat{\mathbf{c}}=\left(\begin{array}{c}
\mathbf{0}_{n \times 1} \\
-\overline{\mathbf{P}} \mathbf{g}_{2}+\mathbf{Z}_{21} B^{-1} v
\end{array}\right) .
\end{aligned}
$$

Considering that each cable only produces pull force, each element in $\mathbf{T}$ of the controller given by (29) must be of positive value. It is impossible to solve $\mathrm{T}$ by (29), because $\widehat{\mathbf{J T}}=M_{o^{\prime \prime}}$ is a scalar and $\mathbf{T}$ is a three-dimensional column vector. Therefore, the additional constraint conditions should be given to successfully solve $\mathbf{T}$ of the controller given by (29); namely, in order to solve the third cable force, the other two cable forces are given. The other two cable forces are chosen as two constant positive values which are convenient to calculate the third cable force. Therefore, the two elements in $\mathbf{T}$ are adopted as two constant positive values and subsequently used to solve the residual element in $\mathbf{T}$ by (29). It depends on how we choose the control gains $C_{p}$ and $C_{d}$ to ensure that the residual element in $\mathbf{T}$ of the controller given by (29) is of positive value. Local asymptotic stability of the closed-loop system is proven by using a Lyapunov stability analysis and an approach similar to that in [22]. 
4.2. Mode Coordinate's Estimation. The controller given in (29) is the function with respect to the flexible spring's modal coordinate variable and the modal velocity variable which are unable to be directly obtained through the sensors. The flexible spring's modal coordinate variable and the modal velocity variable need to be estimated by the measured values of the sensors and then used for calculating the controller given by (29). The spring's first $n$ modes are used for expressing the system's actual response. We denote the flexible spring's modal number to be controlled as $m_{1}$. The spring's first $m_{2}$ modes are estimated where $m_{1}<m_{2}<$ $n$. Hence, we require that the sensors be mounted on the flexible spring's $m_{2}$ points to obtain physical displacements and velocities. The required modal coordinate variable and modal velocity variable of the transverse vibration's $f$ th mode of the flexible spring are gained on the basis of the following equation [23]:

$$
\begin{aligned}
& d_{f}(t)=\sum_{q=1}^{m_{2}}\left(\Pi^{-1}\right)_{f q} w_{l}\left(h_{q}, t\right), \\
& \dot{d}_{f}(t)=\sum_{q=1}^{m_{2}}\left(\Pi^{-1}\right)_{f q} \dot{w}_{l}\left(h_{q}, t\right),
\end{aligned}
$$

where $f=1,2, \ldots, m_{1}$ and $h_{q}$ is the $f$ th sensor's location on the flexible spring for $q=1,2, \ldots, m_{2}, d_{f}(t)$ is the transverse vibration's $f$ th modal coordinate for the flexible spring, $\dot{d}_{f}(t)$ is the transverse vibration's $f$ th modal velocity for the flexible spring, $w_{l}\left(h_{q}, t\right)$ is the transverse deformation of point $h_{q}$ on the flexible spring, and $\dot{w}_{l}\left(h_{q}, t\right)$ is the first derivative of the transverse deformation of point $h_{q}$ on the flexible spring. $w_{l}\left(h_{q}, t\right)=\sum_{f=1}^{n} \delta_{f}(h) d_{f}(t)$ is employed from AMM for the flexible spring. $\left(\Pi^{-1}\right)_{f q}$ in (41) denotes the element of the $f$ th row and the $q$ th column of $\Pi^{-1}$. The transformation matrix $\Pi$ is $m_{2} \times m_{2}$ matrix given by [23]

$$
\Pi=\left(\begin{array}{cccc}
\delta_{1}\left(h_{1}\right) & \delta_{2}\left(h_{1}\right) & \cdots & \delta_{m_{2}}\left(h_{1}\right) \\
\delta_{1}\left(h_{2}\right) & \delta_{2}\left(h_{2}\right) & \cdots & \delta_{m_{2}}\left(h_{2}\right) \\
\vdots & \vdots & \cdots & \vdots \\
\delta_{1}\left(h_{m_{2}}\right) & \delta_{2}\left(h_{m_{2}}\right) & \cdots & \delta_{m_{2}}\left(h_{m_{2}}\right)
\end{array}\right) .
$$

After we obtain the flexible spring's mode coordinates and the mode velocities, the controller given by (29) is able to be calculated. In view of the inconvenience of the sensors' installation, we are unable to obtain the values of $w_{l}\left(h_{q}, t\right)$. Hence, we employ the corresponding open-loop system's simulation values to substitute the values of $w_{l}\left(h_{q}, t\right)$. We only use the transverse vibration's first mode for the flexible spring to denote the response of the system because the flexible spring's length is short.

\section{Simulation and Experiment}

We provide simulation and experimental results to illustrate the practical feasibility of the design for the cable-driven
TABLE 2: Simulation and experimental parameters of the moving platform and the fixed base.

\begin{tabular}{lcc}
\hline$b(\mathrm{~m})$ & $m_{d}(\mathrm{~kg})$ & $a(\mathrm{~m})$ \\
\hline 0.09 & 0.43 & 0.09 \\
\hline
\end{tabular}

TABLE 3: Simulation and experimental parameters of the spring.

\begin{tabular}{lccc}
\hline$\hat{\rho}_{s}(\mathrm{~kg} / \mathrm{m})$ & $L(\mathrm{~m})$ & $E(\mathrm{GPa})$ & $I\left(\mathrm{~m}^{4}\right)$ \\
\hline 1.095 & 0.1016 & 196.5 & $9.811 \times 10^{-12}$ \\
\hline
\end{tabular}

parallel robot and to test the effectiveness of the dynamical model for control and the proposed control scheme in this section. The simulation and experimental parameters of the moving platform and the fixed base are given in Table 2 . The simulation and experimental parameters of the cylindrical compression spring are given in Table 3.

During the flexible spring crooks in the lateral plane, the flexible vibration of $\theta_{s}$ is so small because of the large elastic modulus of the spring. Therefore, our main research is the flexible vibration control of $\theta_{p}$ in the lateral curvature plane. The trajectory tracking control of the center of the moving platform is set as an example to study the effect of the controller given by (29). The center of the moving platform is assumed to move in the lateral curvature plane of $\theta_{s}=210^{\circ}$. The desired trajectory of $\theta$ is given by the following equation:

$$
\begin{aligned}
& \theta_{d} \\
& = \begin{cases}\frac{2 \theta_{i}}{t_{i}^{2}} t^{2}, & t \leq \frac{t_{i}}{2}, \\
\frac{\theta_{i}}{2}+\frac{2 \theta_{i}}{t_{i}}\left(t-\frac{t_{i}}{2}\right)-\frac{2 \theta_{i}}{t_{i}^{2}}\left(t-\frac{t_{i}}{2}\right)^{2}, & \frac{t_{i}}{2}<t \leq t_{i}, \\
\theta_{i}, & t>t_{i} .\end{cases}
\end{aligned}
$$

In (43), we assume that $\theta_{i}=18.6^{\circ}$ and $t_{i}=1.2 \mathrm{~s}$. The desired trajectory of $\theta$ is shown in Figure 8. We control the transverse vibration's first mode for the flexible spring. For the sake of ensuring the control system's stability and all positive cable forces, the control gains $C_{p}$ and $C_{d}$ are selected, respectively, as

$$
\begin{aligned}
& C_{p}=250, \\
& C_{d}=23 .
\end{aligned}
$$

The results of the simulation and the experiment are shown in Figures 9-12.

The system responses under open-loop are shown in Figure 9. Figure 9(a) illustrates the theoretical results of all cable forces without control. Figure 9(b) shows the experimental results of all cable forces without control. Figure 9(c) shows the experimental results of all motor armature currents without control. The image change trend of Figures 9(a) and 9(b) and the image change trend of Figure 9(c) are approximately unanimous. This change rule is consistent with the theoretical derivation given by (6). Figures 9 (b) and 9(c) have the phenomena of burr which are caused by the sensors noises. Figure 9(d) shows the theoretical transverse 


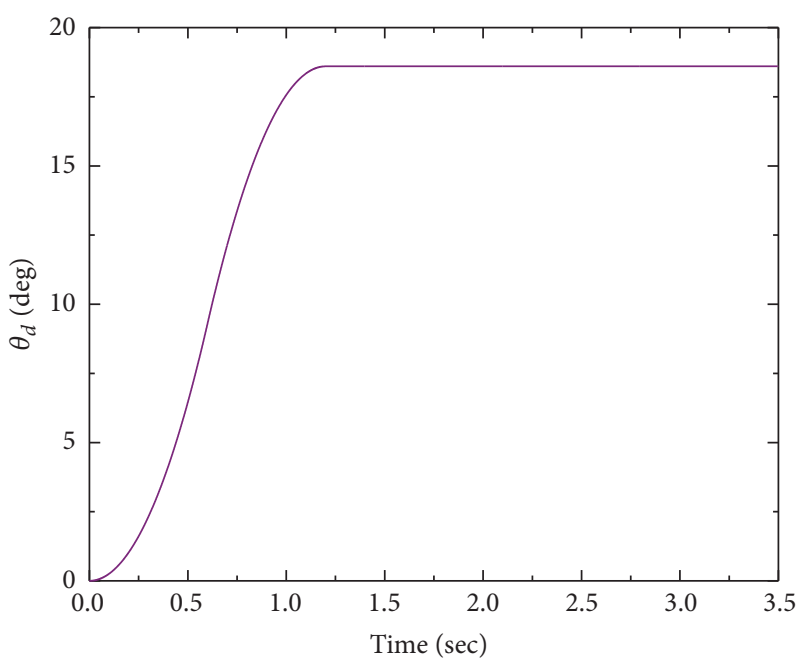

Figure 8: Desired trajectory of $\theta$.

deformation displacement of $o$ point without control. Figures 9(e) and 9(f) show the theoretical and experimental results of $\theta_{p}$ without control. As seen from Figures 9(e) and 9(f), $\theta_{p}$ performs a wide range of movement while maintaining the vibration because the spring is flexible without control.

The system responses under closed-loop are shown in Figure 10. Figure 10(a) illustrates the theoretical results of all cable forces with control. Figure 10(b) shows the experimental results of all cable forces with control. Figure 10(c) illustrates the experimental results of all motor armature currents with control. The image change trend of Figures 10(a) and 10(b) and the image change trend of Figure 10(c) are approximately unanimous. This change rule matches the theoretical derivation given in (6). Figures 10(b) and 10(c) have the phenomena of burr which are caused by the sensors noises. Figure 10(d) shows the experimental results of all cable lengths with control. From Figure 10(d), we can observe the following rules: $l_{2}$ shortens its length when $l_{1}$ and $l_{3}$ stretch their lengths; $l_{2}$ stretches its length when $l_{1}$ and $l_{3}$ shorten their lengths. These features fit the objective law. As can be seen from Figures 10(e) and 10(f), the controller given by (29) can successfully reduce the vibration of the flexible spring.

Figure 11 illustrates the variation results of $\theta_{s}$. As can be seen from Figure 11, the experimental vibration of $\theta_{s}$ is small during the flexible spring's lateral curvature plane movement. Figure 12 shows a comparison of $\theta_{p}$ values for the desired trajectory, the simulation, and the experiment during the lateral curvature plane movement. As we can observe from Figure 12, the controller given by (29) meets a relatively good performance in the arrival index of the desired position and the desired time for the center of the moving platform.

It can be concluded that we validate the robot prototype, the theoretical model for control, and the proposed control method by the simulation and experiment results.

\section{Conclusions and Future Work}

This paper carries out the mechanical design and electrical design of the cable-driven parallel robot. Meanwhile, the theoretical and experimental studies on the vibration control of the center of the moving platform for the trajectory tracking movement are executed. First, we elaborate a cabledriven parallel robot device that can efficiently be applied to the experimental research. Then, the dynamic model of the system is derived by AMM and Lagrange's equation. After that, system nonlinear controller is designed to control the spring-induced vibration of the moving platform's trajectory tracking. Eventually, we provide the simulation study and analyze the experiment result of the vibration control to validate the usability of the cable-driven parallel robot prototype and to verify the proposed control scheme. The approximate consistency for the vibration control results of the simulation and experiment in Section 5 verifies the feasibility of the designed cable-driven parallel robot system and the proposed control project. In addition, the proposed nonlinear controller can be applied in other similar flexible parallel systems.

Based on the study of the design and control of a 2-DOF flexible parallel humanoid arm joint robot, our future work will focus on the experimental study of antivibration control of the whole humanoid arm system.

\section{Appendix}

$$
\begin{aligned}
M_{11} & =\frac{1}{3} \widehat{\rho}_{s} L^{3}+m_{d} h^{2}+m_{d} \mathbf{d}^{\mathrm{T}}\left(\delta^{\mathrm{T}} \delta\right) \mathbf{d} \\
& +\mathbf{d}^{\mathrm{T}}\left(\hat{\rho}_{s} \int_{0}^{L} \delta^{\mathrm{T}} \delta \mathrm{d} h\right) \mathbf{d}, \\
\mathbf{M}_{12} & =\widehat{\rho}_{s} \int_{0}^{L} h \delta \mathrm{d} h+m_{d} h \delta, \\
\mathbf{M}_{21} & =2 \dot{\theta}\left(\hat{\rho}_{s} \int_{0}^{L} \delta^{\mathrm{T}} \delta \mathrm{d} h\right) \dot{\mathbf{d}}+\hat{\rho}_{s} \int_{0}^{L} h \delta^{\mathrm{T}} \mathrm{d} h \\
& +m_{d} h \delta^{\mathrm{T}}, \\
\mathbf{M}_{22} & =\dot{\theta}^{2} \hat{\rho}_{s} \int_{0}^{L} \delta^{\mathrm{T}} \delta \mathrm{d} h+\hat{\rho}_{s} \int_{0}^{L} \delta^{\mathrm{T}} \delta \mathrm{d} h+m_{d} \delta^{\mathrm{T}} \delta, \\
H_{1} & =2 \dot{\theta} m_{d} \mathbf{d}^{\mathrm{T}}\left(\delta^{\mathrm{T}} \delta\right) \dot{\mathbf{d}}+2 \dot{\theta} \mathbf{d}^{\mathrm{T}}\left(\hat{\rho}_{s} \int_{0}^{L} \delta^{\mathrm{T}} \delta \mathrm{d} h\right) \dot{\mathbf{d}}, \\
\mathbf{K}_{22} & =E I \int_{0}^{L} \ddot{\delta} \ddot{\delta} \mathrm{d} h-\dot{\theta}^{2} \hat{\rho}_{s} \int_{0}^{L} \delta^{\mathrm{T}} \delta \mathrm{d} h-m_{d} \dot{\theta}^{2} \delta^{\mathrm{T}} \delta, \\
\mathbf{H}_{2} & =\mathbf{0}_{n \times 1}, \\
K_{11} & =0, \\
\mathbf{K}_{12} & =\mathbf{0}_{1 \times n}, \\
\mathbf{K}_{21} & =\mathbf{0}_{n \times 1}, \\
&
\end{aligned}
$$



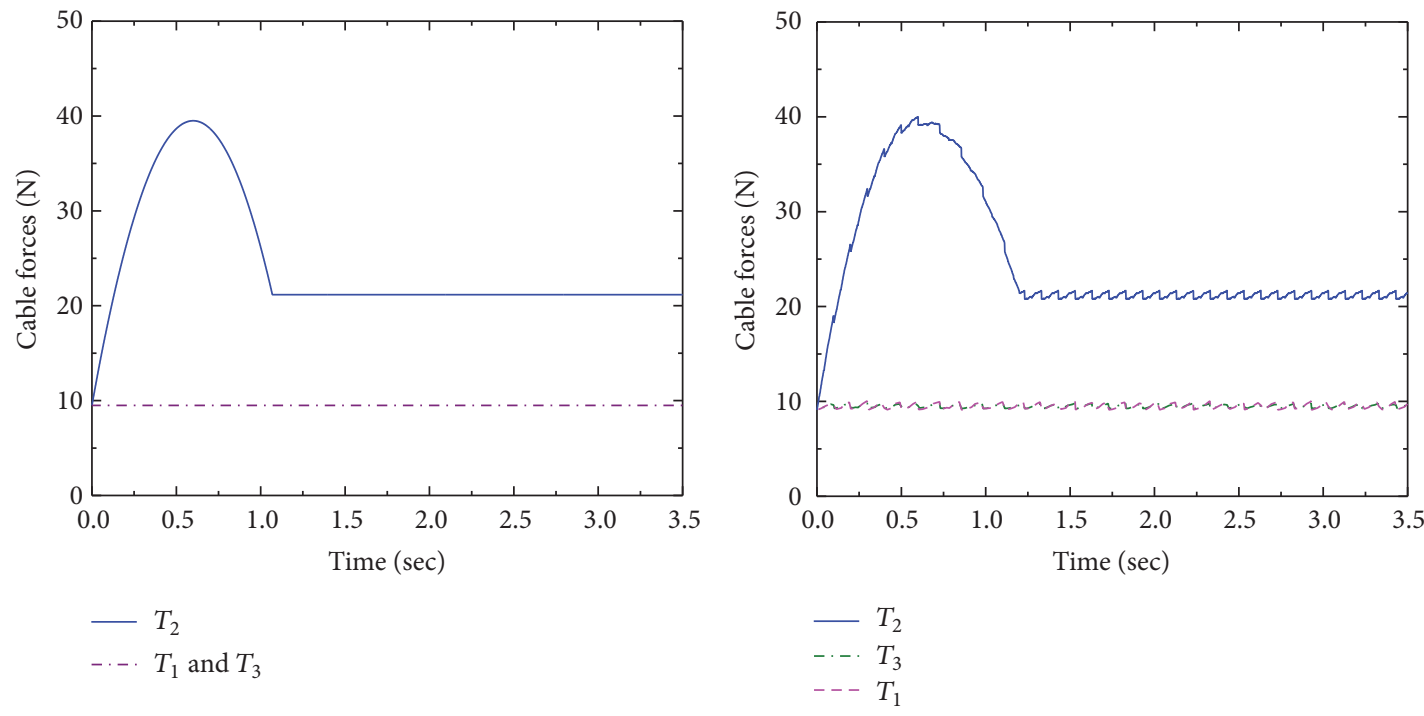

(a)

(b)
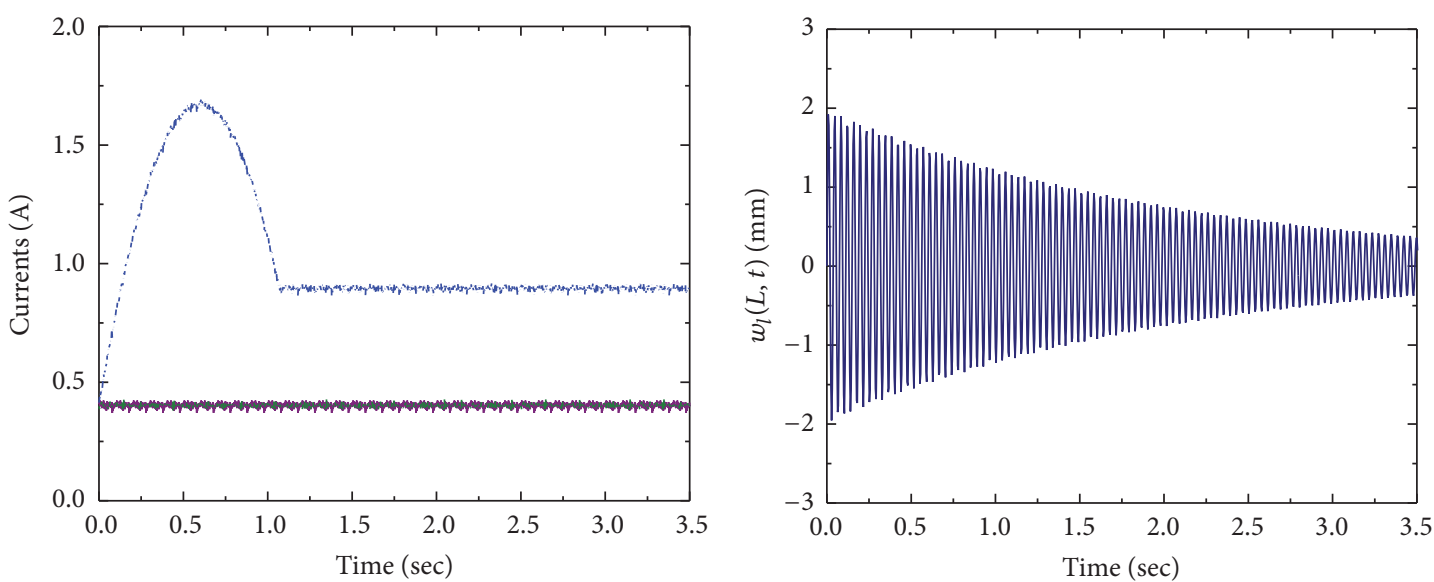

- Motor $1\left(T_{1}\right)$

- - Motor $3\left(T_{3}\right)$

-.. Motor $2\left(T_{2}\right)$

(c)

(d)

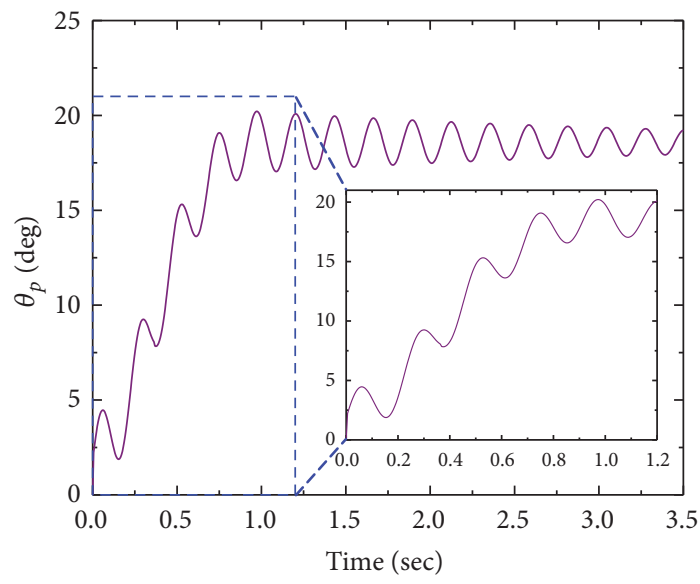

(e)

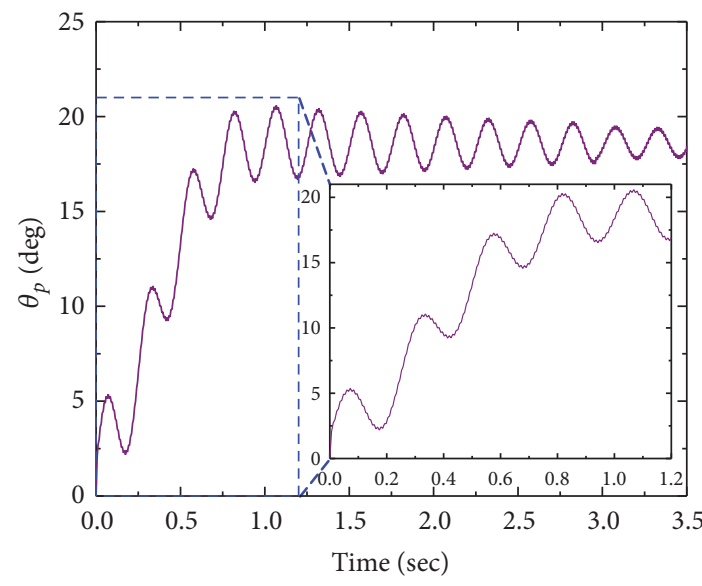

(f)

FIGURE 9: Responses of the system under open-loop: (a) theoretical results of all cable forces under open-loop, (b) experimental results of all cable forces under open-loop, (c) experimental results of all motor armature currents under open-loop, (d) theoretical transverse deformation result of the point $o$ under open-loop, (e) theoretical result of $\theta_{p}$ under open-loop, and (f) experimental result of $\theta_{p}$ under open-loop. 

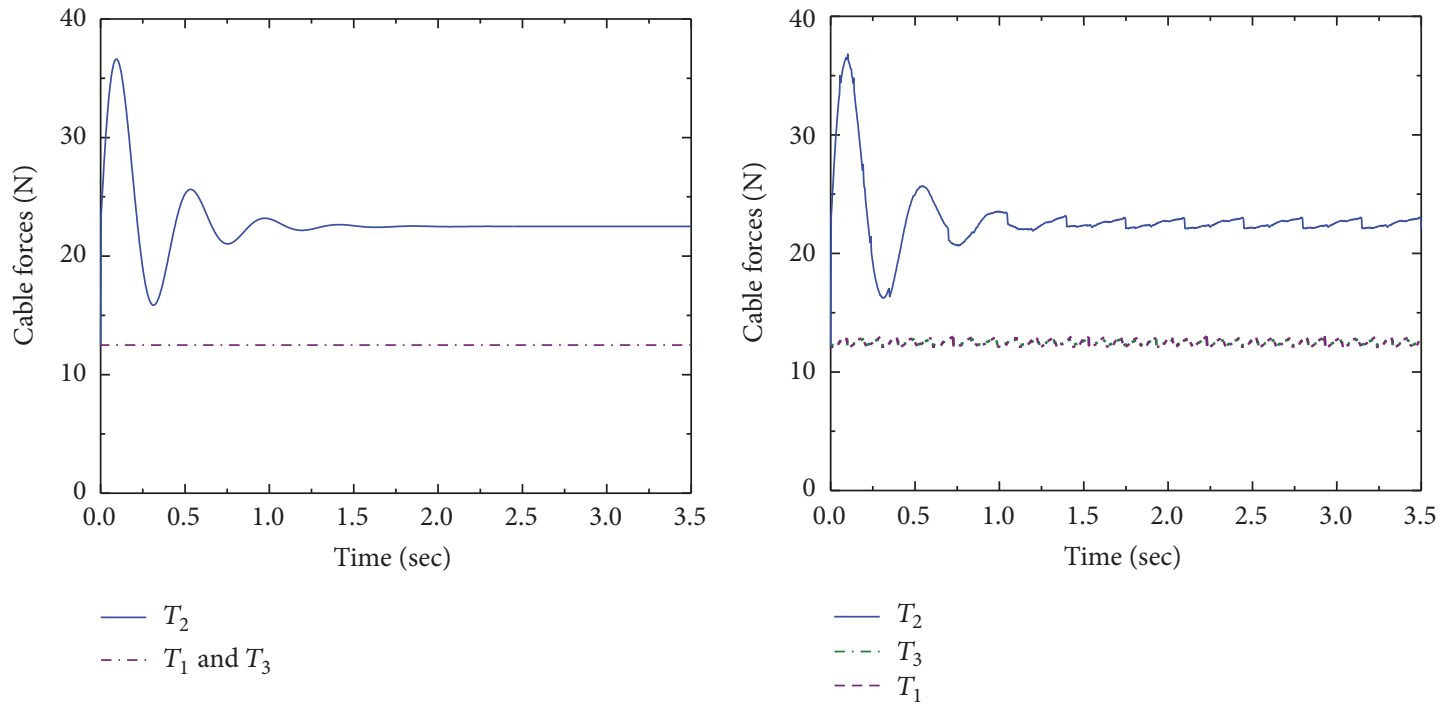

(a)

(b)
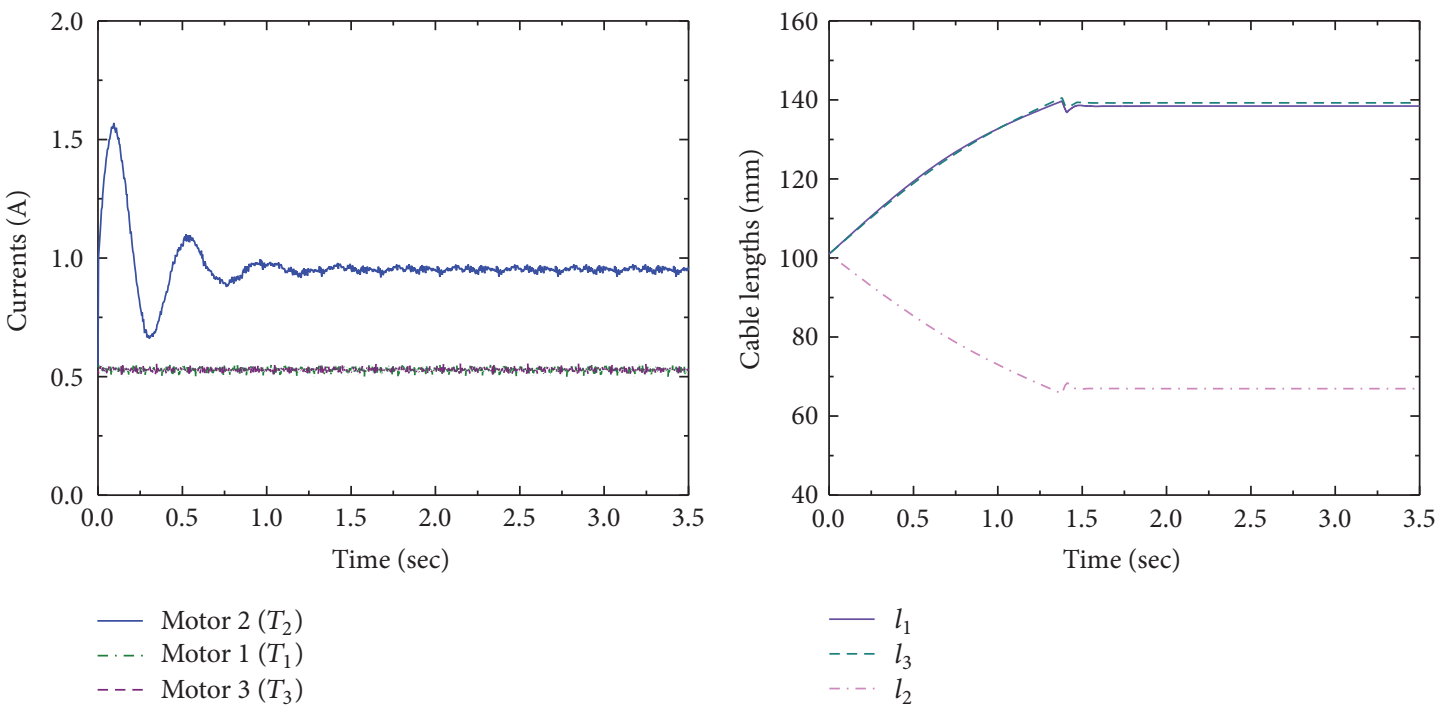

(c)

(d)

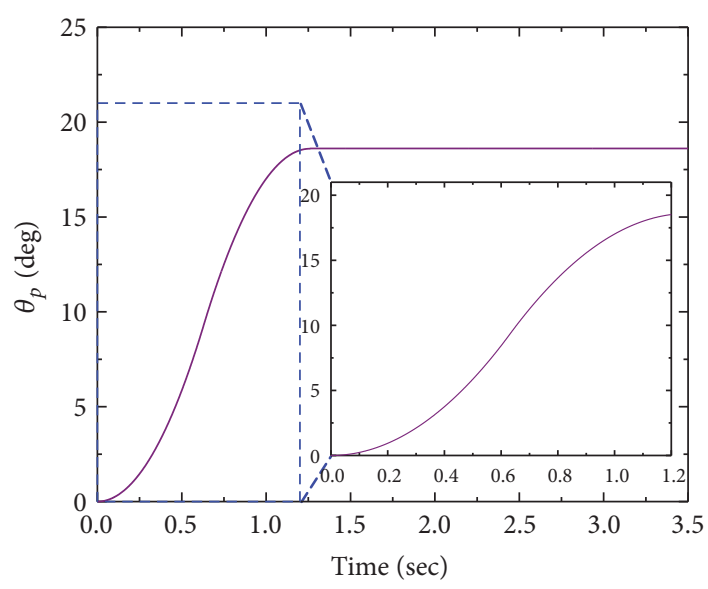

(e)

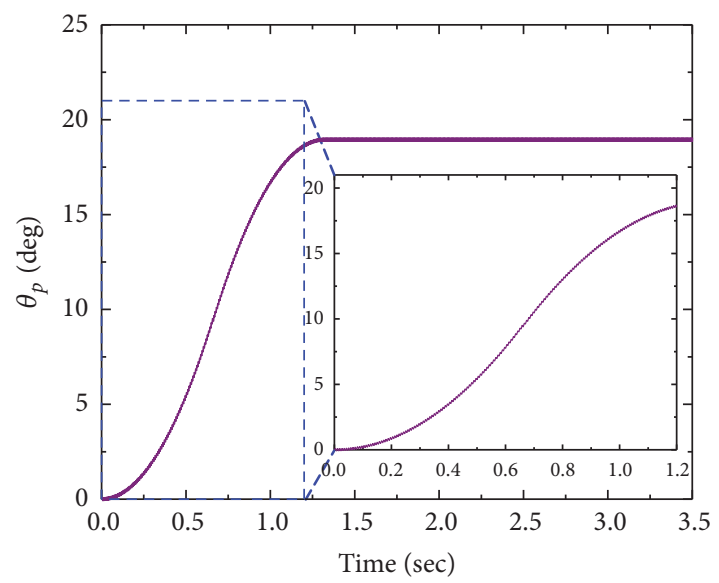

(f)

FIGURE 10: Responses of the system under closed-loop: (a) theoretical results of all cable forces under closed-loop, (b) experimental results of all cable forces under closed-loop, (c) experimental results of all motor armature currents under closed-loop, (d) experimental results of all cable lengths under closed-loop, (e) theoretical result of $\theta_{p}$ under closed-loop, and (f) experimental result of $\theta_{p}$ under closed-loop. 


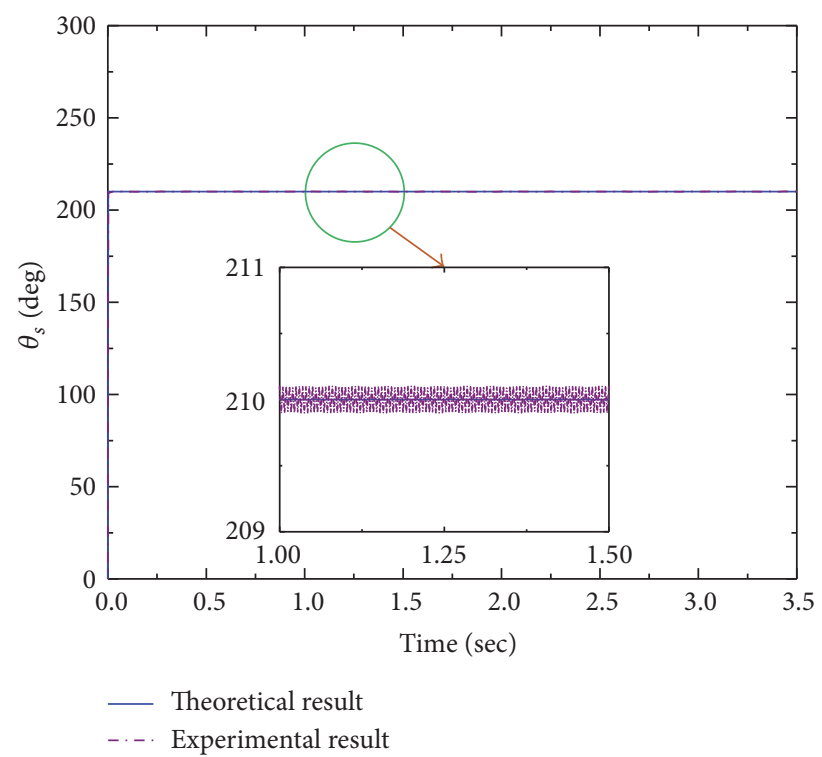

Figure 11: Variation results of $\theta_{s}$.

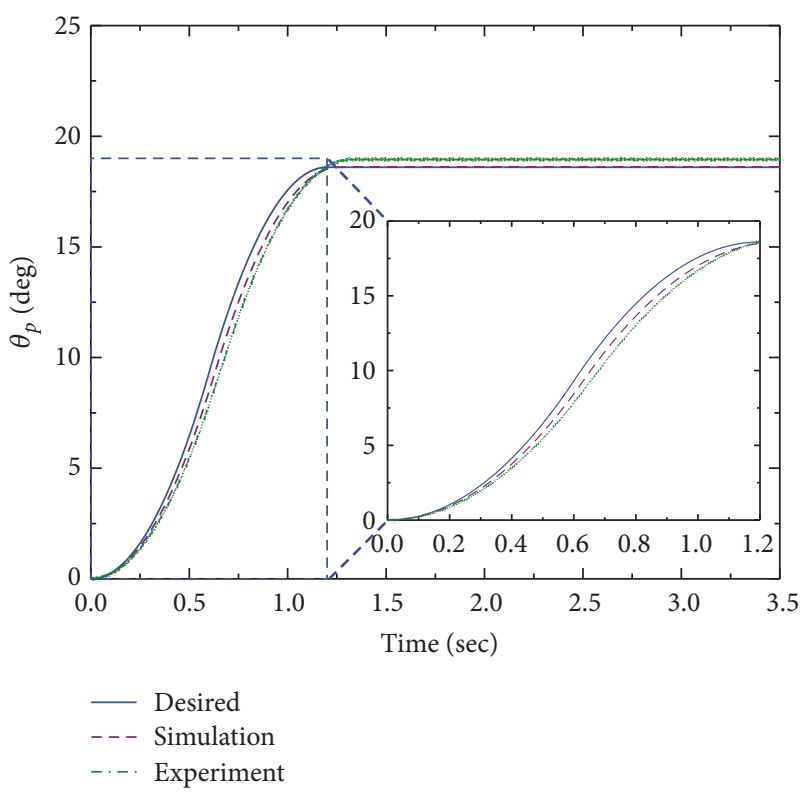

FIGURE 12: Variation results of $\theta_{p}$.

$$
\mathbf{G}_{2}=\mathbf{0}_{n \times 1}
$$$$
\widehat{J_{1}}=\frac{1}{s \theta_{s}}\left(b \bar{z} p_{1} c \alpha+a b p_{1} s \alpha c \beta-b \bar{y} p_{1} s \alpha c \beta+a p_{1} \bar{e}_{1}\right.
$$$$
\left.-b p_{1} \bar{e}_{1} c \alpha-\bar{y} p_{1} \bar{e}_{1}\right)+b p_{1} \bar{e}_{2} s \alpha c \beta+\bar{z} p_{1} \bar{e}_{2}
$$$$
\widehat{J}_{2}=\frac{1}{s \theta_{s}}\left(-\frac{1}{2} b \bar{z} p_{2} c \alpha-\frac{\sqrt{3}}{4} a b p_{2} s \beta-\frac{\sqrt{3}}{2} b \bar{y} p_{2} s \beta\right.
$$$$
+\frac{1}{4} a b p_{2} s \alpha c \beta+\frac{1}{4} b \bar{y} p_{2} s \alpha c \beta-\frac{1}{2} a p_{2} \bar{e}_{1}
$$

$$
\begin{aligned}
& \left.+\frac{1}{2} b p_{2} \bar{e}_{1} c \alpha-\bar{y} p_{2} \bar{e}_{1}\right)+\frac{\sqrt{3}}{2} b p_{2} \bar{e}_{2} s \beta-\frac{1}{2} \\
& \cdot b p_{2} \bar{e}_{2} s \alpha c \beta+\bar{z} p_{2} \bar{e}_{2} \\
& \widehat{J_{3}}=\frac{1}{s \theta_{s}}\left(-\frac{1}{2} b \bar{z} p_{3} c \alpha+\frac{\sqrt{3}}{4} a b p_{3} s \beta+\frac{\sqrt{3}}{2} b \bar{y} p_{3} s \beta\right. \\
& +\frac{1}{4} a b p_{3} s \alpha c \beta+\frac{1}{2} b \bar{y} p_{3} s \alpha c \beta-\frac{1}{2} a p_{3} \bar{e}_{1} \\
& \left.+\frac{1}{2} b p_{3} \bar{e}_{1} c \alpha-\bar{y} p_{3} \bar{e}_{1}\right)-\frac{\sqrt{3}}{2} b p_{3} \bar{e}_{2} s \beta-\frac{1}{2} \\
& \cdot b p_{3} \bar{e}_{2} s \alpha c \beta+\bar{z} p_{3} \bar{e}_{2} \text {, } \\
& p_{1}=\frac{1}{\sqrt{w_{1}}} \\
& w_{1}=(b s \alpha s \beta+\bar{x})^{2}+(a-b c \alpha-\bar{y})^{2}+(b s \alpha c \beta+\bar{z})^{2} \text {, } \\
& p_{2}=\frac{1}{\sqrt{w_{2}}} \\
& w_{2}=\left(\frac{\sqrt{3}}{2} a-\frac{\sqrt{3}}{2} b c \beta-\frac{1}{2} b s \alpha s \beta+\bar{x}\right)^{2}+\left(\frac{1}{2} a\right. \\
& \left.-\frac{1}{2} b c \alpha+\bar{y}\right)^{2}+\left(\frac{\sqrt{3}}{2} b s \beta-\frac{1}{2} b s \alpha c \beta+\bar{z}\right)^{2} \text {, } \\
& p_{3}=\frac{1}{\sqrt{w_{3}}} \\
& w_{3}=\left(\frac{\sqrt{3}}{2} a-\frac{\sqrt{3}}{2} b c \beta+\frac{1}{2} b s \alpha s \beta-\bar{x}\right)^{2}+\left(\frac{1}{2} a\right. \\
& \left.-\frac{1}{2} b c \alpha+\bar{y}\right)^{2}+\left(\frac{\sqrt{3}}{2} b s \beta+\frac{1}{2} b s \alpha c \beta-\bar{z}\right)^{2}, \\
& s \theta_{s}=\sin \theta_{s}, \\
& s \alpha=\sin \alpha, \\
& c \alpha=\cos \alpha, \\
& s \beta=\sin \beta \text {, } \\
& c \beta=\cos \beta \text {. }
\end{aligned}
$$

\section{Conflicts of Interest}

The authors declare that there are no conflicts of interest regarding the publication of this paper.

\section{Acknowledgments}

This study was supported by the Excellent Young Teachers Program of Southeast University (2242015R30024) and Six Talent Peaks Project of Jiangsu Province (2014-ZBZZ-001). 


\section{References}

[1] J.-Y. Park, B.-H. Cho, S.-H. Byun, and J.-K. Lee, "Development of cleaning robot system for live-line suspension insulator strings," International Journal of Control, Automation and Systems, vol. 7, no. 2, pp. 211-220, 2009.

[2] G. Yang, W. Lin, M. S. Kurbanhusen et al., "Kinematic design of a 7-DOF cable-driven humanoid arm: a solution-in-nature approach," in Proceedings of the IEEE/ASME International Conference on Advanced Intelligent Mechatronics, pp. 24-28, Monterey, Calif, USA, July 2005.

[3] Q. Boehler, S. Abdelaziz, M. Vedrines, P. Poignet, and P. Renaud, "From modeling to control of a variable stiffness device based on a cable-driven tensegrity mechanism," Mechanism and Machine Theory, vol. 107, pp. 1-12, 2017.

[4] N. Zhang and W. Shang, "Dynamic trajectory planning of a 3-DOF under-constrained cable-driven parallel robot," Mechanism and Machine Theory, vol. 98, pp. 21-35, 2016.

[5] J. Albus, R. Bostelman, and N. Dagalakis, "The NIST robocrane," Journal of National Institute of Standards and Technology, vol. 97, no. 3, pp. 373-385, 1992.

[6] L. L. Cone, "Skycam-an aerial robotic camera system," Byte, vol. 10 , no. 10 , pp. 122-132, 1985.

[7] J. Du, H. Bao, and C. Cui, "Stiffness and dexterous performances optimization of large workspace cable-driven parallel manipulators," Advanced Robotics, vol. 28, no. 3, pp. 187-196, 2014.

[8] X. Tang and Z. Shao, "Trajectory generation and tracking control of a multi-level hybrid support manipulator in FAST," Mechatronics, vol. 23, no. 8, pp. 1113-1122, 2013.

[9] Y. Yang and W. Zhang, "An elephant-trunk manipulator with twisting flexional rods," in Proceedings of the IEEE International Conference on Robotics and Biomimetics, pp. 13-18, Zhuhai, China, December 2015.

[10] C. Laschi, M. Cianchetti, B. Mazzolai, L. Margheri, M. Follador, and P. Dario, "Soft robot arm inspired by the octopus," Advanced Robotics, vol. 26, no. 7, pp. 709-727, 2012.

[11] D. Rus and M. T. Tolley, "Design, fabrication and control of soft robots," Nature, vol. 521, no. 7553, pp. 467-475, 2015.

[12] J. Du, H. Bao, C. Cui, and D. Yang, "Dynamic analysis of cabledriven parallel manipulators with time-varying cable lengths," Finite Elements in Analysis and Design, vol. 48, no. 1, pp. 13921399, 2012.

[13] S.-R. Oh and S. K. Agrawal, "A reference governor-based controller for a cable robot under input constraints," IEEE Transactions on Control Systems Technology, vol. 13, no. 4, pp. 639-645, 2005.

[14] B. Gao, J. Xu, J. Zhao, and N. Xi, "Combined inverse kinematic and static analysis and optimal design of a cable-driven mechanism with a Spring Spine," Advanced Robotics, vol. 26, no. 8-9, pp. 923-946, 2012.

[15] C. Lusty, N. Sahinkaya, and P. Keogh, "A novel twin-shaft rotor layout with active magnetic couplings for vibration control," Proceedings of the Institution of Mechanical Engineers. Part I: Journal of Systems and Control Engineering, vol. 230, no. 3, pp. 266-276, 2016.

[16] X. Y. Yu and L. Chen, "Singular perturbation adaptive control and vibration suppression of free-flying flexible space manipulators," Proceedings of the Institution of Mechanical Engineers Part C: Journal of Mechanical Engineering Science, vol. 229, no. 11, pp. 1989-1997, 2015.

[17] D. X. Phu, N. V. Quoc, J.-H. Park, and S.-B. Choi, "Design of a novel adaptive fuzzy sliding mode controller and application for vibration control of magnetorheological mount," Proceedings of the Institution of Mechanical Engineers Part C: Journal of Mechanical Engineering Science, vol. 228, no. 13, pp. 2285-2302, 2014.

[18] Z. Mohamed and M. O. Tokhi, "Vibration control of a singlelink flexible manipulator using command shaping techniques," Proceedings of the Institution of Mechanical Engineers. Part I: Journal of Systems and Control Engineering, vol. 216, no. 2, pp. 191-210, 2002.

[19] M. Moallem, R. V. Patel, and K. Khorasani, "An inverse dynamics control strategy for tip position tracking of flexible multi-link manipulators," Journal of Field Robotics, vol. 14, no. 9, pp. 649-658, 1997.

[20] B. Gao, H. Song, J. Zhao, S. Guo, L. Sun, and Y. Tang, "Inverse kinematics and workspace analysis of a cable-driven parallel robot with a spring spine," Mechanism and Machine Theory, vol. 76, pp. 56-69, 2014.

[21] S. Lu, B. Gao, Z. Zhu, and Y. Tang, "Vibration analysis of a bioinspired flexible parallel mechanism," in Proceedings of the IEEE International Conference on Cyber Technology in Automation, Control and Intelligent Systems, pp. 630-635, Shenyang, China, June 2015.

[22] M. Moallem, R. V. Patel, and K. Khorasani, "Nonlinear tipposition tracking control of a flexible-link manipulator: Theory and experiments," Automatica, vol. 37, no. 11, pp. 1825-1834, 2001.

[23] D.-A. Wang and Y.-M. Huang, "Application of discrete-time variable structure control in the vibration reduction of a flexible structure," Journal of Sound and Vibration, vol. 261, no. 3, pp. 483-501, 2003. 


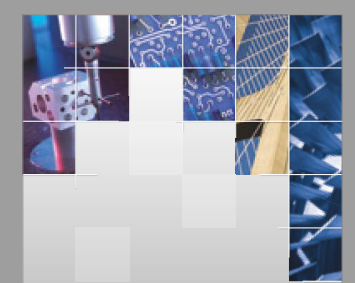

\section{Enfincering}
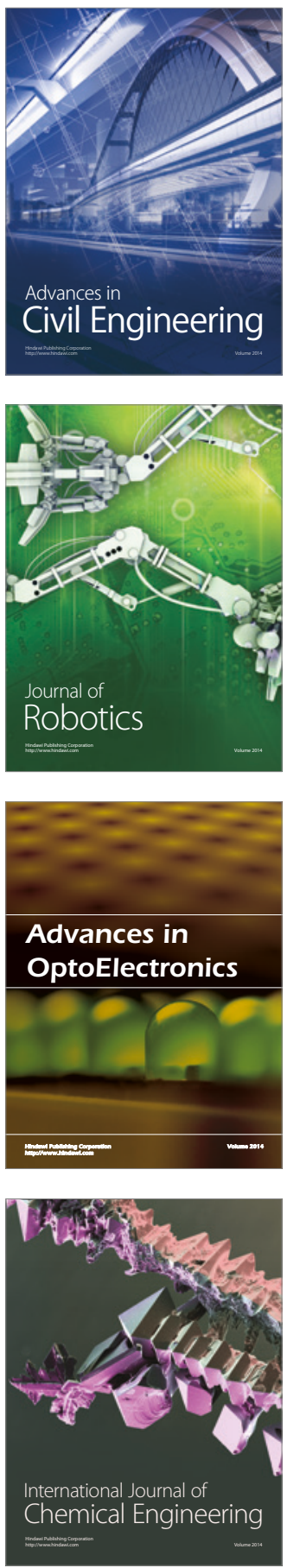

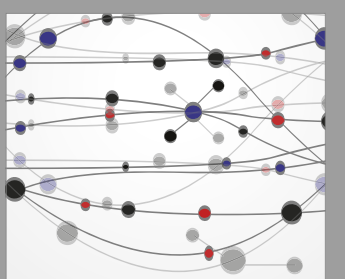

The Scientific World Journal

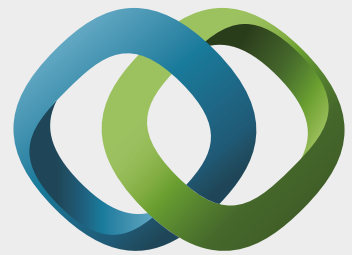

\section{Hindawi}

Submit your manuscripts at

https://www.hindawi.com
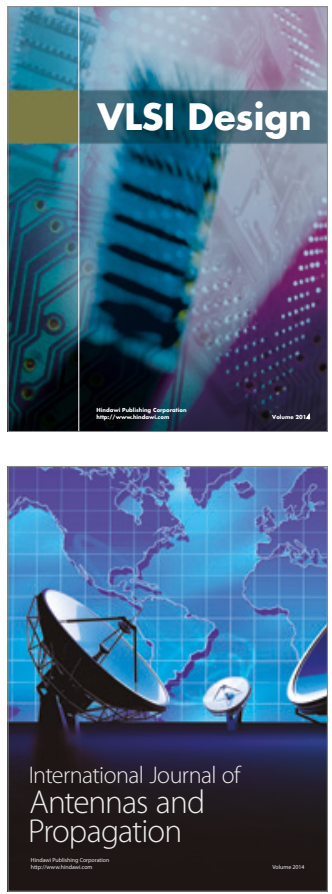

\section{Rotating}

Machinery
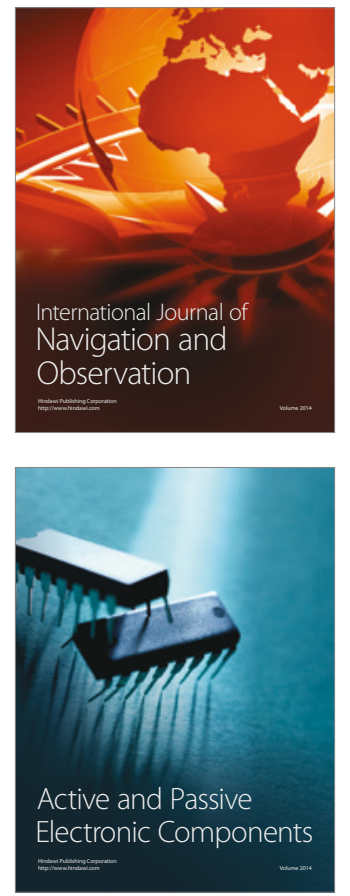
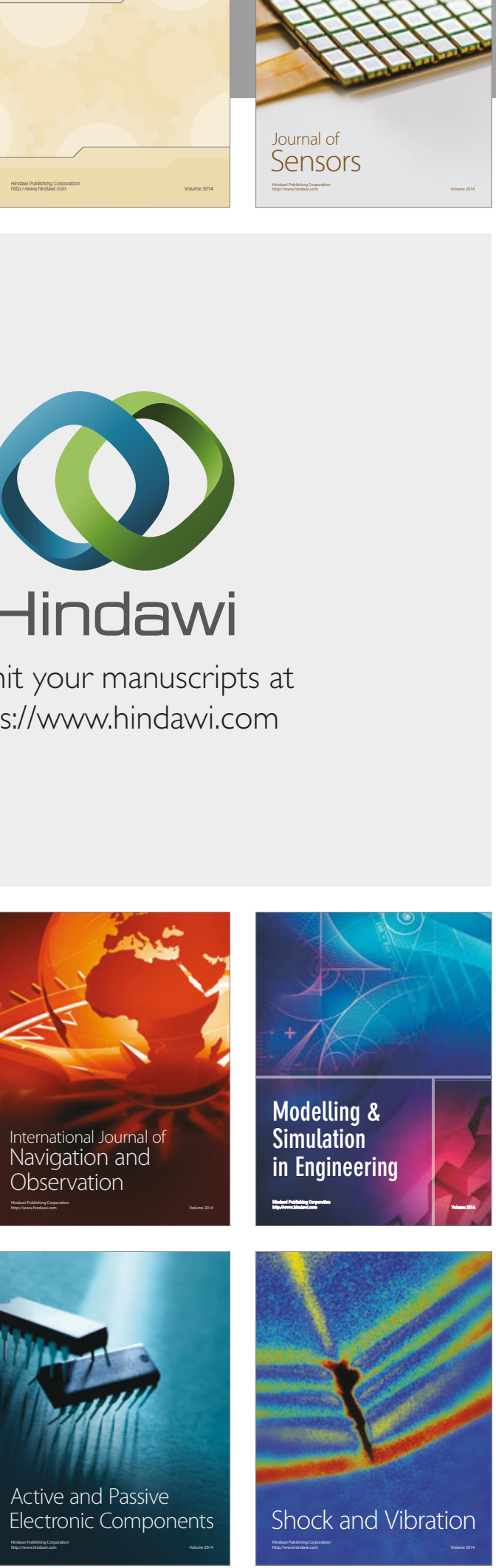
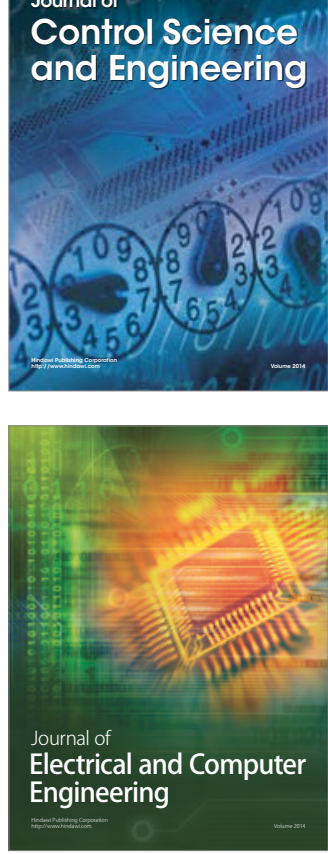

Distributed

Journal of

Control Science

and Engineering
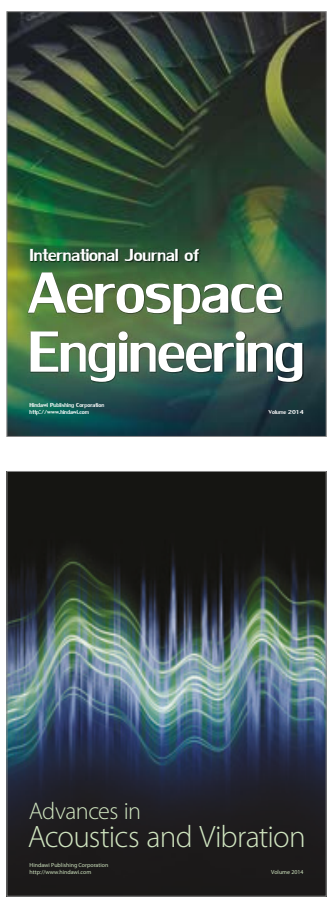

Sensor Networks 